\title{
The ROK-family regulator Rok7B7 directly controls carbon catabolite repression, antibiotic biosynthesis, and morphological development in Streptomyces avermitilis
}

\author{
Xiaorui Lu, ${ }^{1}$ Xingchao Liu, ${ }^{1}$ Zhi Chen ${ }^{10},{ }^{1}$ Jilun $\mathrm{Li}^{1}{ }^{1}$ \\ Gilles P. van Wezel, ${ }^{2}$ Wei Chen $^{3 *}$ and Ying Wen $\mathbb{1}^{1 *}$ \\ ${ }^{1}$ State Key Laboratory of Agrobiotechnology and \\ College of Biological Sciences, China Agricultural \\ University, Beijing, China. \\ ${ }^{2}$ Molecular Biotechnology, Institute of Biology, Leiden \\ University, Leiden, The Netherlands. \\ ${ }^{3}$ Clinical Research Center, the Second Hospital of \\ Nanjing, Nanjing University of Chinese Medicine, \\ Nanjing, China.
}

\section{Summary}

Carbon catabolite repression (CCR) is a common phenomenon in bacteria that modulates expression of genes involved in uptake of alternative carbon sources. In the filamentous streptomycetes, which produce half of all known antibiotics, the precise mechanism of CCR is yet unknown. We report here that the ROK-family regulator Rok7B7 pleiotropically controls xylose and glucose uptake, CCR, development, as well as production of the macrolide antibiotics avermectin and oligomycin A in Streptomyces avermitilis. Rok7B7 directly repressed structural genes for avermectin biosynthesis, whereas it activated olmRl, the cluster-situated activator gene for oligomycin A biosynthesis. Rok7B7 also directly repressed the xylose uptake operon xyIFGH, whose expression was induced by xylose and repressed by glucose. Both xylose and glucose served as Rok7B7 ligands. rok7B7 deletion led to enhancement and reduction of avermectin and oligomycin A production, respectively, relieved CCR of xyIFGH, and increased co-uptake efficiency of xylose and glucose. A consensus Rok7B7-binding site, 5'-TTKAMKHSTTSAV-3',

Received 8 February, 2020; revised 10 May, 2020; accepted 19 May, 2020. *For correspondence. E-mail njyy039@njucm.edu.cn; Tel. (+86) 2585091648 . E-mail wen@ @au.edu.cn; Tel. (+86) 1062732715 . [Correction added on 03 July 2020 after first online publication: The affiliation of the $5^{\text {th }}$ and $6^{\text {th }}$ author has been corrected in this version.] was identified within aveA1p, olmRIp, and xyIFp, which allowed prediction of the Rok7B7 regulon and confirmation of 11 additional targets involved in development, secondary metabolism, glucose uptake, and primary metabolic processes. Our findings will facilitate methods for strain improvement, antibiotic overproduction, and co-uptake of xylose and glucose in Streptomyces species.

\section{Introduction}

The Gram-positive bacterial streptomycetes is known for two unique traits: (i) production of a wide variety of medically useful antibiotics and (ii) a complex life cycle involving the successive formation of vegetative hyphae, aerial hyphae, and chains of spores (Flardh and Buttner, 2009; van Wezel and McDowall, 2011). Carbon catabolite repression (CCR), a process occurring in streptomycetes and other bacteria that ensures the preferred uptake of carbon source (e.g., glucose) despite the presence of alternative ones in the environment. CCR also plays a role in the control of development and antibiotic production in streptomycetes where glucose is usually the driver of this process (van Wezel and McDowall, 2011; RomeroRodriguez et al., 2017). In streptomycetes, glucose is transported through the major facilitator superfamily (MFS) transporter GlcP and phosphorylated by glucose kinase (GIkA) and not through the phosphoenolpyruvatedependent phosphotransferase system (PTS) (van Wezel et al., 2005). GlkA plays a role in CCR (van Wezel and McDowall, 2011; Romero-Rodriguez et al., 2017) even if it lacks a DNA-binding domain. The way GlkA exerts its regulatory role is unknown. In summary, our knowledge regarding regulation of $\mathrm{CCR}$ in streptomycetes at the molecular level is very poor, despite many decades of research.

At least 20 families of transcriptional regulators (TRs) have been discovered in streptomycetes, including TetR, LuxR, MarR, AraC, LysR, GntR, SARP, and ROK (Repressor, Open Reading Frame, Kinase) (RomeroRodriguez et al., 2015). The ROK-family regulators have 
a characteristic PF00480 motif and are widely distributed in prokaryotes, but the specificity and function for most of them remain unknown (Kazanov et al., 2013). ROKfamily regulators characterized so far are involved mainly in sugar metabolism; e.g., Mlc and NagC in E. coli regulate uptake of glucose and $\mathrm{N}$-acetylglucosamine (GICNAc) respectively (Plumbridge, 2001). The best-studied ROKfamily regulator in streptomycetes is S. lividans CsnR that auto-regulates negatively its own expression as well as that of a chitosanase encoding gene in response to products of chitosan degradation (Dubeau et al., 2011). In S. coelicolor, Rok7B7 (SCO6008) is involved in the control of antibiotic production, development, xylose uptake, and CCR (Swiatek et al., 2013), but its specific DNAbinding sites and ligands have not been identified. In this strain, another ROK-family regulator, RokB (SCO6115) acts as a direct regulator of heterologously expressed novobiocin biosynthetic gene cluster (BGC). The predicted RokB regulon suggests involvement of RokB in transport and metabolism of amino acids, but not of sugars (Bekiesch et al., 2016). Furthermore, the effect of deletion of genes encoding other ROK-family regulators, SCO0794, SCO1060, SCO2846, SCO7543, SCO6566, or SCO6600 on the development and antibiotic production of S. coelicolor was reported (Swiatek et al., 2013). Therefore, the manipulation of regulatory networks governed by ROK-family regulators might be beneficial for strain improvement and thus deserves deeper investigation.

The industrially important species $S$. avermitilis is a well-known producer of avermectins, which are economically potent anthelmintic antibiotics widely applied in agricultural and medical fields (Burg et al., 1979; Egerton et al., 1979). Streptomyces avermitilis also produces oligomycin A, a 26-membered macrolide antibiotic that displays antifungal and antitumor activities (Pinna et al., 1967; Lin et al., 2009). The 82-kb avermectin BGC contains one cluster-situated regulator (CSR) gene, ave $R$, that encodes a LuxR-family activator essential for expression of ave structural genes (Guo et al., 2010; Kitani et al., 2009). The $\sim 100-\mathrm{kb}$ oligomycin BGC contains two CSR genes, olmRI and olmRII, that encode
LuxR-family activators essential for oligomycin biosynthesis (Yu et al., 2012). The S. avermitilis genome encodes 17 putative ROK-family regulators (including Rok7B7 orthologue SAV_2248), of which none has been studied so far.

In the present study, we characterized $S$. avermitilis Rok7B7 as a dual repressor/activator in development, avermectin and oligomycin A production, xylose and glucose uptake, and other primary metabolic processes. Rok7B7 targets associated with these processes were identified. Both xylose and glucose act as ligands of Rok7B7 and its orthologues from model S. coelicolor and $S$. venezuelae, but they play opposing roles in modulating its DNA-binding activity. Repression of the xylose uptake operon $x y / F G H$ by glucose-induced $\mathrm{CCR}$ is dependent on Rok7B7. We also described a novel strategy for increasing avermectin yield and improving couptake efficiency of xylose and glucose through deletion of rok7B7 gene.

\section{Results}

Rok7B7 affects development and biosynthesis of avermectins and oligomycin $A$

The gene rok7B7 (sav_2248) from S. avermitilis consists of 1200 nucleotides (nt) and encodes a 399-amino acid protein. Protein alignment showed a high conservation, with $91.2 \%, 90 \%, 94 \%, 94.3 \%$ and $94.3 \%$ overall amino acid identity to its orthologs in S. griseus, S. venezuelae, $S$. scabies, S. lividans, and $S$. coelicolor respectively. rok7B7 is located upstream of the $x y / F G H$ operon (sav_2247, sav_2246, sav_2245) that encodes a xylose $A B C$ transport system (Swiatek et al., 2013) and is transcribed in the same direction as the latter (Fig. 1). $x y / G$ overlaps $x y l H$ with $4 \mathrm{bp}$, and the intergenic regions $x y I F-x y / G$ and rok7B7-xylF are 184 and 123 bp long respectively (Fig. 1). RT-PCR analysis revealed that $x y / F$ is co-transcribed with $x y / G$, whereas rok7B7 is not co-transcribed with $x y I F$ (Supporting Information Fig. S1), indicating that $x y I F, x y I G$, and $x y l H$ form an operon and rok7B7 has its own promoter.

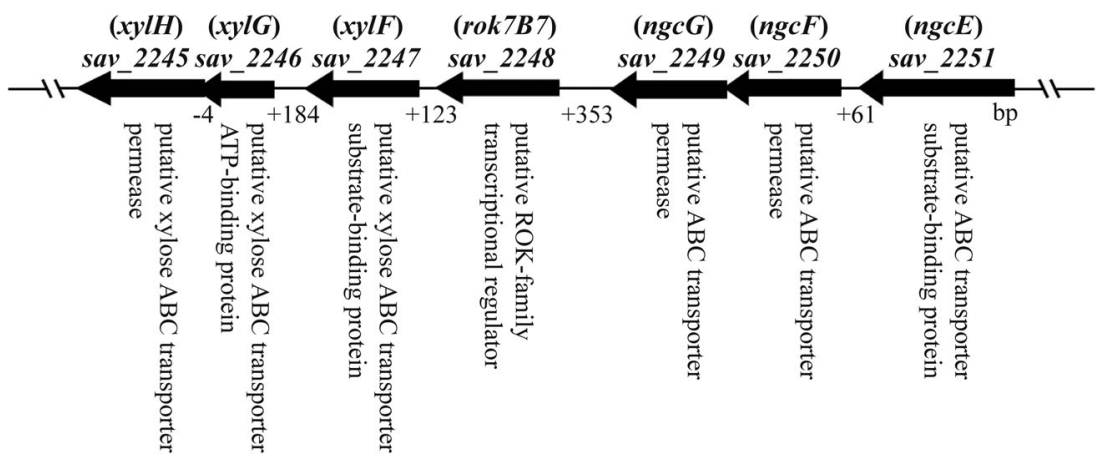

Fig. 1. Genetic organization of rok7B7 and its neighbouring genes. 
To investigate the role of Rok7B7 in S. avermitilis, we constructed a mutant strain with an in-frame deletion of rok7B7 ( $\triangle$ rok7B7) (Supporting Information Fig. S2), this mutant complemented with a single copy of rok7B7 (Crok7B7) and a strain, which overexpresses rok7B7 (Orok7B7). qRT-PCR analyses revealed that rok7B7 transcription was undetectable in $\triangle$ rok7B7, and that its level in Orok7B7 was $\sim 1.6$-fold higher on day 2 (exponential phase) and $\sim 5$.4-fold higher on day 6 (stationary phase) than that in the wild-type (WT) strain (Supporting Information Fig. S3), confirming deletion or overexpression of rok7B7 in the strains.

$\Delta$ rok7B7, Crok7B7, and Orok7B7 were grown on solid YMS sporulation medium for phenotypic comparison with WT. $\Delta$ rok7B7 displayed earlier differentiation and sporulation, whereas Orok7B7 and Crok7B7 were similar to WT (Fig. 2A). Faster differentiation of $\triangle$ rok7B7 was confirmed by scanning electron microscopy (SEM) of samples grown on YMS plates for 2 or 4 days (Fig. 2B). On day 2, WT colonies mainly consisted of vegetative mycelia, whereas $\Delta$ rok7B7 colonies were already sporulated. On day 4 , both strains formed spore chains. These findings indicate an inhibitory role of Rok7B7 in S. avermitilis development.

We then investigated the effects of deletion and overexpression of rok7B7 on growth and antibiotic biosynthesis. Quantitative analysis by HPLC of the fermentation broth from 10-day cultures grown in FM-I showed that, relative to WT level, avermectin yield was increased by $\sim 106 \%$ in $\Delta$ rok7B7, but was reduced by $\sim 46 \%$ in Orok7B7. Avermectin production of the complemented mutant Crok7B7 and of control strains (WT/pKC1139,
WT/pSET152) was nearly identical to that of WT (Fig. 3A). Oligomycin A yield was reduced by $\sim 38 \%$ in $\Delta$ rok7B7 and increased by $\sim 78 \%$ in Orok7B7 relative to WT level (Fig. 3B). Biomass accumulation of $\Delta$ rok7B7 and Orok7B7 grown in soluble FM-II was similar to that of WT (Fig. 3C), indicating that altered antibiotic yields of $\Delta$ rok7B7 and Orok7B7 were not due to changes of cell growth. These findings indicate that Rok7B7 differentially regulates production of avermectins and oligomycin $A$.

In view of the significant increase of avermectin yield resulting from the deletion of rok7B7, we also deleted the gene in industrial strain 63\#, using the same strategy as for WT, and examined the effect on avermectin yield. In shake-flasks, avermectin yield was $\sim 13 \%-16 \%$ higher for $\Delta$ rok7B7/63\# than for 63\# (Fig. 3D). Although in this case, the deletion of rok7B7 does not seem to have had much effect in the industrial strain, compared with the WT, it is a potentially efficient strategy to enhance avermectin production in industrial strains.

\section{Rok7B7 represses avermectin structural genes and activates the gene for oligomycin CSR}

Transcription of rok7B7 in WT grown in FM-I was assessed by qRT-PCR to further establish the role of Rok7B7 in the control of antibiotic production in $S$. avermitilis. rok7B7 transcript levels reached a maximum after 2 days of growth, followed by a gradual decrease, reaching low levels after 6 days (Fig. 4A). Simultaneously, Rok7B7 protein levels were examined by western blotting. Expression of fusion protein Rok7B7-3FLAG in $\Delta$ rok7B7 (strain $\Delta$ rok7B7/rok7B7-3FLAG) restored avermectin production
A

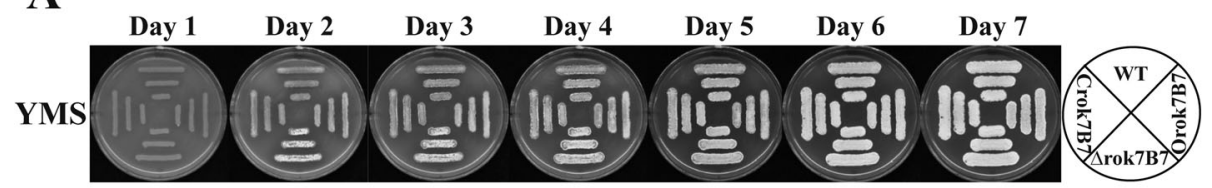

B

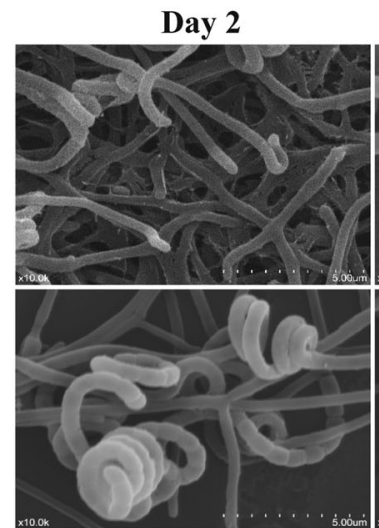

$\times 10.0 \mathrm{k}$

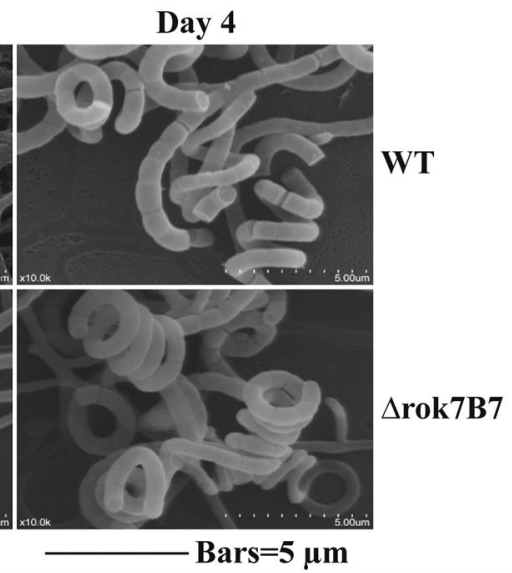

Fig. 2. Effect of Rok7B7 on S. avermitilis development.

A. Phenotypes of WT strain, rok7B7 deletion mutant ( $\triangle$ rok7B7), complemented strain (Crok7B7), and overexpression strain (Orok7B7) grown on YMS plates at $28^{\circ} \mathrm{C}$.

B. SEM images of WT and $\triangle$ rok7B7 after growth on YMS plates for 2 or 4 days. 
A

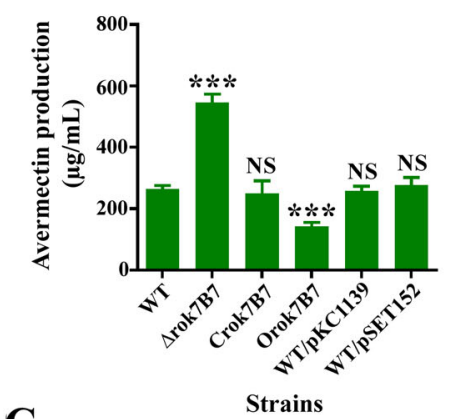

C

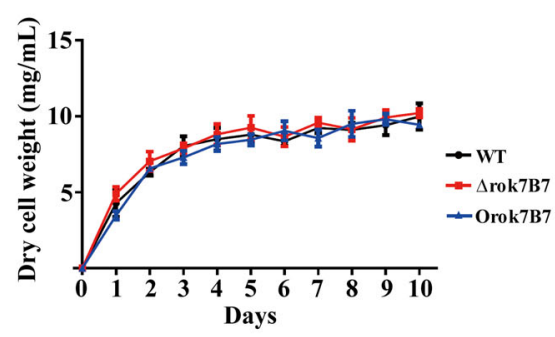

B

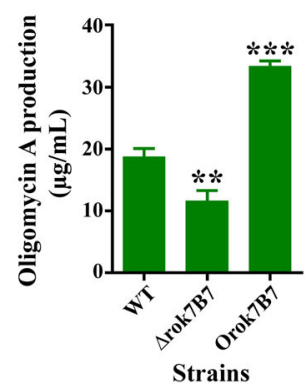

D

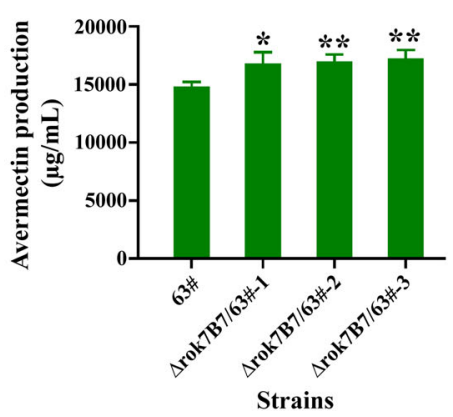

Fig. 3. Effects of Rok7B7 on antibiotic production and cell growth in $S$. avermitilis.

$A$ and $B$. Yield of avermectins (A) and oligomycin $A(B)$ in rok7B7-related strains cultured in FM-I for 10 days. WT/pSET152 and WT/pKC1139 are the empty vector control strains.

C. Growth curves of WT, $\triangle$ rok7B7, and Orok7B7 cultured in FM-II.

D. Avermectin yield in industrial strain 63\# and its derivatives $\Delta$ rok7B7/63\#-1, -2 , and -3 (rok7B7 deletion strains) grown in FM-I for 10 days. Error bars (all panels): SD from triplicate experiments. Statistical notations (panels A, B, D): NS, not significant; ${ }^{\star} P<0.05$; ${ }^{\star \star} P<$ $0.01 ;{ }^{* \star \star} P<0.001$ for comparison with WT $(\mathrm{A}, \mathrm{B})$ or 63\# (D) (Student's $t$-test). [Color figure can be viewed at wileyonlinelibrary.com] to WT level (Supporting Information Fig. S4), indicating that Rok7B7-3FLAG complemented Rok7B7 function, and that Rok7B7 expression profile could be monitored with anti-FLAG antibody against Rok7B7-3FLAG in $\Delta$ rok7B7/rok7B7-3FLAG. Consistent with the transcriptional profile, Rok7B7 protein level was maximal after 2 days, low after 6 days and barely detectable from day 7 onward (Fig. 4B). These findings indicate that Rok7B7 plays its role particularly toward the end of exponential phase.

To determine whether Rok7B7 regulates avermectin and oligomycin A production directly through CSR genes or structural genes, we performed electrophoretic mobility shift assays (EMSAs) using soluble $\mathrm{His}_{6}$-tagged Rok7B7 purified from E. coli. Probes corresponding to the promoter regions of three CSR genes, namely aveRp, olmRIp, and olmRIlp, were used for EMSAs (Fig. 4C). The ave gene cluster contains four genes for polyketide synthase (PKS), namely aveA1, aveA2, aveA3, and aveA4, which synthesize the avermectin polyketide backbone. ave $A 1$ and ave $A 2$ are cotranscribed, and ave $A 4$ and ave $A 3$ are co-transcribed (lkeda et al., 1999). The seven PKS genes in the olm gene cluster form two transcriptional units: olmA1-olmA2olmA3-olmA6-olmA7 and olmA4-olmA5 (Omura et al., 2001). Accordingly, we designed promoter probes aveA1p (for aveA1-aveA2), aveA4p (for aveA4-aveA3), olmA1p (for olmA1-olmA2-olmA3-olmA6-olmA7), and olmA4p (for olmA4-olmA5) for EMSA probes of structural genes (Fig. 4C). As control we used the probe hrdBp, corresponding to the promoter region of $h r d B$, which encodes the principal RNA polymerase $\sigma$ factor $\mathrm{HrdB}$.
$\mathrm{His}_{6}$-Rok7B7 bound specifically to probes aveA1p and olmRIp, but not to aveRp, aveA4p, olmRIlp, olmA1p, or olmA4p, nor to the negative control $h r d B p$ (Fig. 4D). Binding specificities were confirmed by competition assays using $\sim 300$-fold unlabeled specific probes (lanes S) or nonspecific probe $h r d B p$ (lanes N). aveA2 is co-transcribed with ave $A 1$, and is therefore also a target of Rok7B7.

To confirm in vivo binding of Rok7B7 to target promoters of aveA1 and olmRl, we performed chromatin immunoprecipitation-quantitative PCR (ChIP-qPCR) assays using samples from WT and $\Delta$ rok7B7/rok7B73FLAG cultured in soluble FM-II for 1-6 days, and antiFLAG mAb against Rok7B7-3FLAG. WT without Rok7B7-3FLAG was used as negative control. aveRp did not display Rok7B7 enrichment (Fig. 4E), confirming that ave $R$ is indirectly regulated by Rok7B7. In contrast, Rok7B7 bound to aveA1p and olmRlp at various time points; binding was strongest at day 2 and decreased thereafter (Fig. 4E), consistent with the expression profile of Rok7B7. These findings confirm dynamic binding of Rok7B7 to the target promoters aveA1p and olmRlp in vivo.

The effect of Rok7B7 on the expression of target genes aveA1, aveA2, and olmRl was assessed by qRTPCR. RNA samples were isolated after 2 and 6 days from WT and $\triangle$ rok7B7 grown in FM-I. aveA1 and aveA2 transcription levels were higher in $\triangle$ rok7B7 at both time points, whereas olmRI level in $\triangle$ rok7B7 was lower, consistent with the antibiotic yield data for $\Delta$ rok7B7 (Fig. 4F).

These experiments revealed an interesting difference in the avermectin and oligomycin $A$ production 
controlled by Rok7B7: Rok7B7 interacted with the promoter regions of the ave structural genes but not with that of the activator gene aveR, while, in contrast, Rok7B7 interacted with the promoter region of the activator gene of oligomycin biosynthetic cluster.

\section{Determination of Rok7B7-binding sites}

Understanding the regulatory mechanism of Rok7B7 on its target promoters requires determination of precise Rok7B7-binding sites. Despite many such efforts using DNase I footprinting assays, we were unable to detect
A

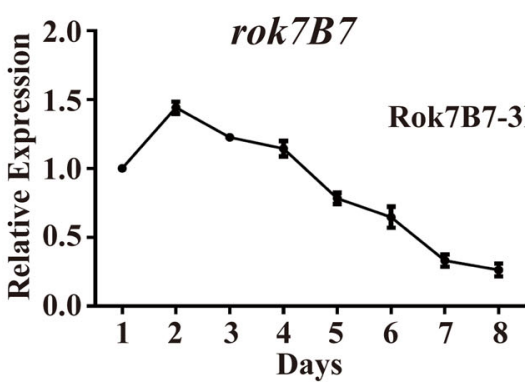

B $\begin{array}{lllllllll}\text { Days } & 1 & 2 & 3 & 4 & 5 & 6 & 7 & 8\end{array}$

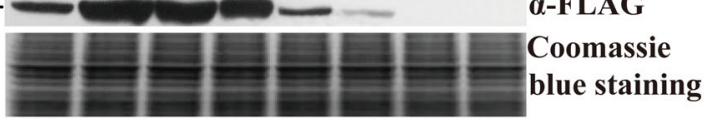

C

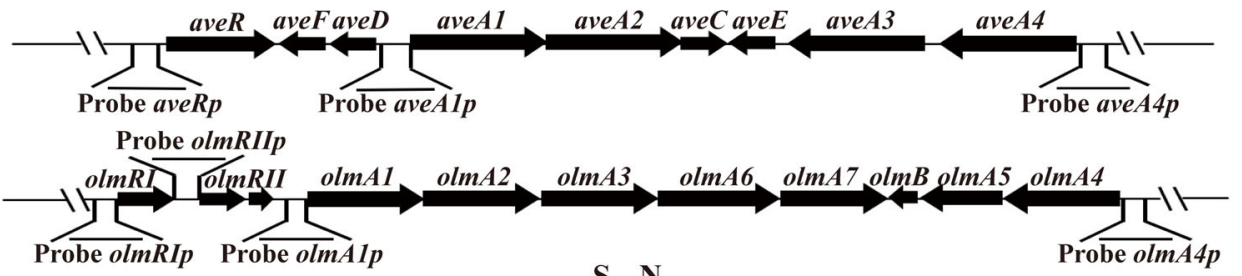

D
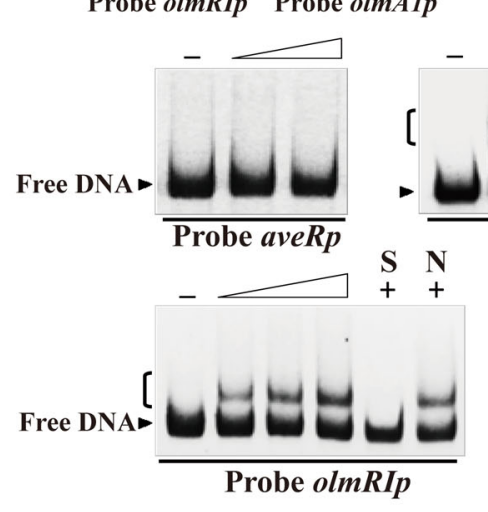

$\begin{array}{ll}\mathbf{S} & \mathbf{N} \\ + & +\end{array}$
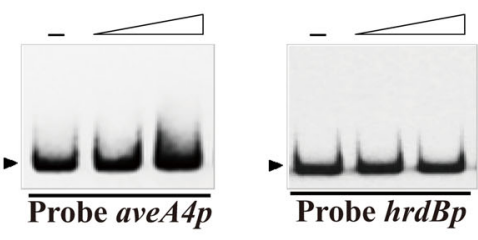

Probe aveAtp
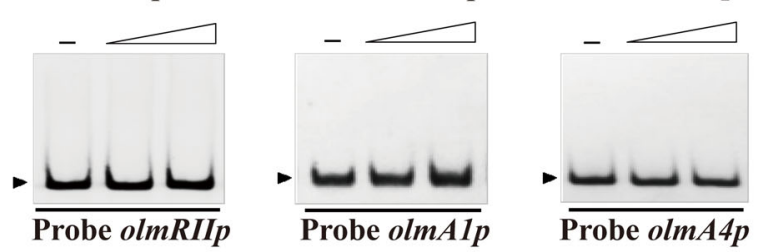

$\mathbf{E}$
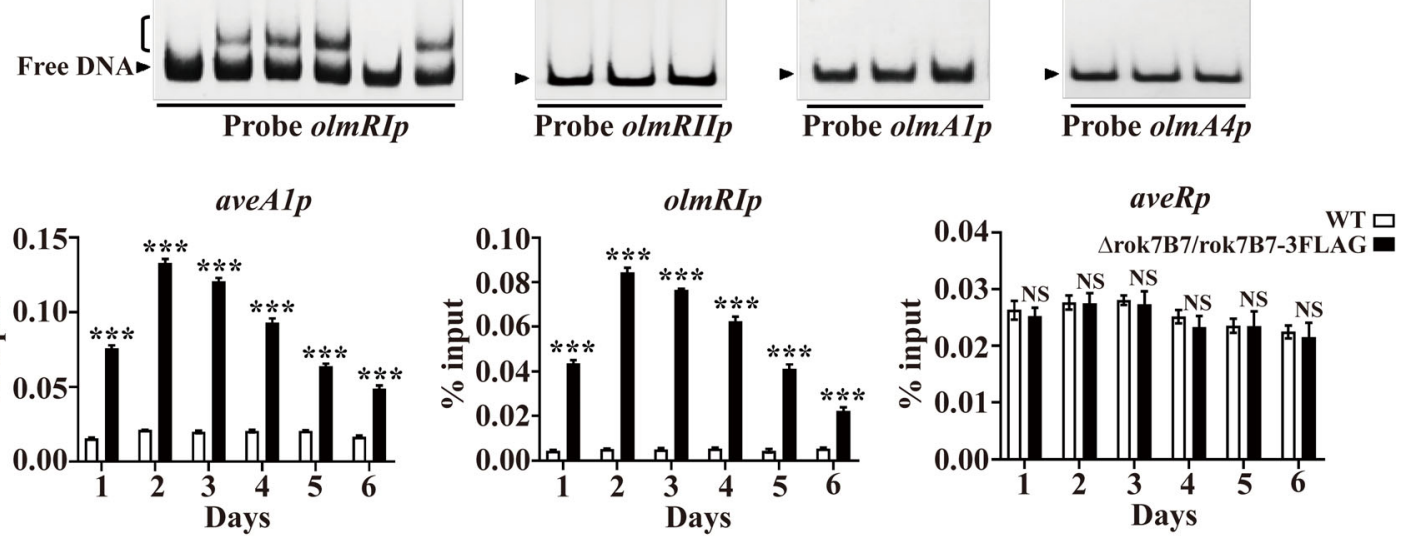

F
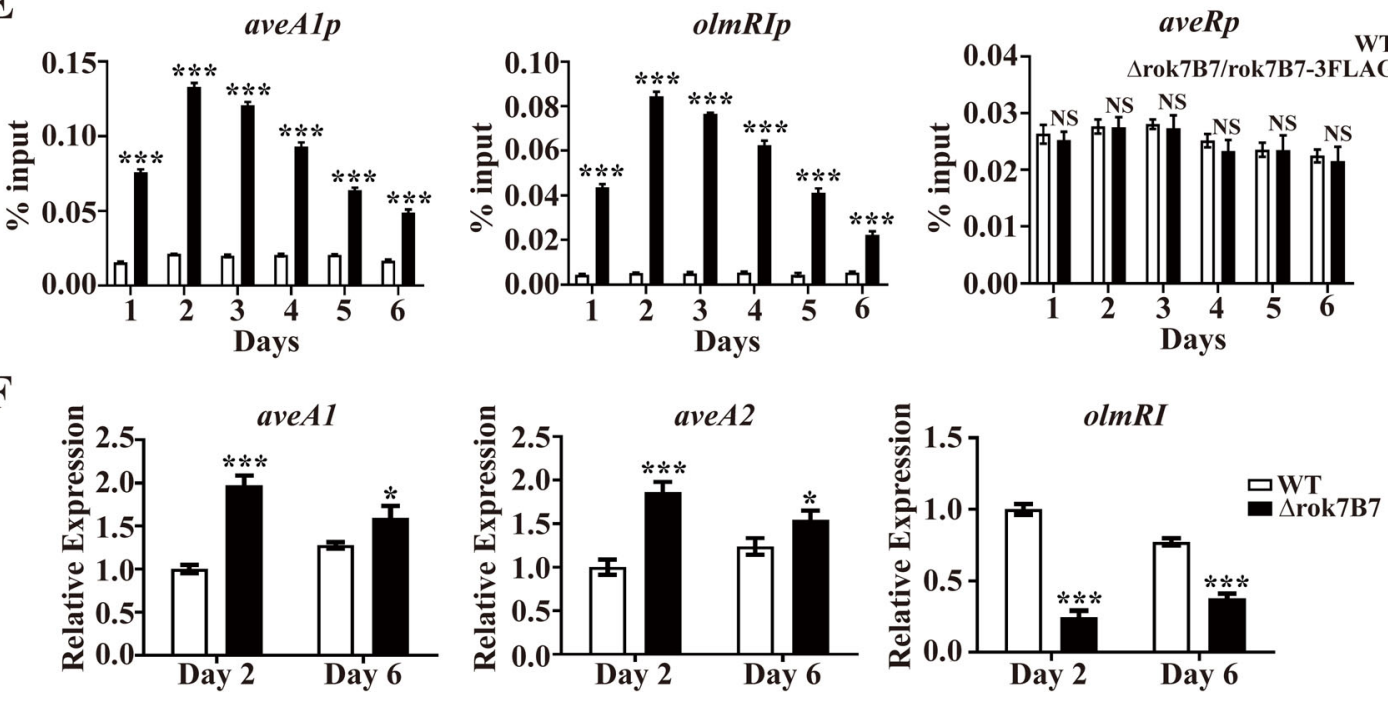

Fig. 4. Legend on next page. 
Rok7B7-binding sites on promoter regions of any of the identified target genes, most likely because of the low DNA-binding activity of Rok7B7. We therefore performed EMSAs, using a series of overlapping probes, to identify the protected site of Rok7B7 on aveA1p. Overlapping probes aveA1p-I and aveA1p-II covered the entire 303-bp aveA1p probe, and a 108-bp overlapping sequence was designated as probe aveA1p-III (Fig. 5A). $\mathrm{His}_{6}-$ Rok7B7 bound to probes aveA1p-I, aveA1p-II, and aveA1p-III (Fig. 5A), indicating that the Rok7B7-binding site(s) on aveA1p are located within the 108-bp overlapping region.

DNAMAN analysis of the 108-bp sequence of probe aveA1p-III revealed a direct repeat sequence (TTGAA GAGTTGAA; termed $D R^{\text {ave }}$ ) and a palindromic sequence (TTGAAGACGTTCAA; termed $I^{\text {ave }}$ ). To shorten the Rok7B7 binding region, we designed a 50-bp probe aveA1p-IV containing both sequences $D^{\text {ave }}$ and $\mathrm{IR}^{\text {ave }}$. EMSAs of $\mathrm{His}_{6}-$ Rok7B7/probe aveA1p-IV interaction revealed a band shift, suggesting that $D R^{\text {ave }}$ or $\mathbb{I R}^{\text {ave }}$ may serve as target site for Rok7B7 binding. To evaluate relative contributions of $\mathrm{DR}^{\text {ave }}$ and $\mathrm{IR}^{\text {ave }}$ in Rok7B7 binding, we introduced mutations into repeat motifs of probe aveA1p-IV to generate a series of 50-bp mutated probes (Fig. 5B). His $_{6}$-Rok7B7 bound to probe $3 \mathrm{~m}$ but not to $1 \mathrm{~m}, 2 \mathrm{~m}$, or $4 \mathrm{~m}$ (Fig. 5B), indicating that the 5-bp direct repeats in sequence $D^{\text {ave }}$, but not inverted repeats in $\mathbb{I R}^{\text {ave }}$, are essential for Rok7B7 binding.

As TRs typically bind to similar DNA motifs in target promoter regions, we searched and found an imperfect direct repeat sequence (TTGACTCGTTCAG; termed DR ${ }^{\text {olm }}$ ) similar to $\mathrm{DR}^{\text {ave }}$ in probe olmR/p. The ability of Rok7B7 to bind to $\mathrm{DR}^{\mathrm{olm}}$ was evaluated by EMSAs using 50 -bp probes containing either intact sequence $\mathrm{DR}^{\mathrm{olm}}$ (termed probe olmR/pI) or the mutated sequence lacking direct repeats (termed probe $5 \mathrm{~m}$ ) (Fig. $5 \mathrm{C}$ ). Affinity of $\mathrm{His}_{6}-$ Rok7B7 for the mutated probe $5 \mathrm{~m}$ was abolished in comparison with corresponding WT probe olmRIp-I (Fig. 5C), indicating that the direct repeats in $D^{\text {olm }}$ play an essential role in Rok7B7 binding.
Binding kinetics of Rok7B7 with 50-bp probes aveA1p-IV and olmR/p-I, respectively containing Rok7B7-binding sites $D^{\text {ave }}$ and $D R^{\text {olm }}$, were studied by biolayer interferometry (BLI) assay. The sensorgrams revealed a direct correlation of binding strength with $\mathrm{His}_{6}$-Rok7B7 concentration. The equilibrium dissociation constants $\left(K_{D}\right)$ for interaction with Rok7B7 were 31.7 and $13.9 \mathrm{nM}$ for aveA1p-IV and for olmR/p-l, respectively (Fig. 5D). Thus, DNA affinity of Rok7B7 was much lower than that of CsnR, the ROK-family regulator from $S$. lividans $\left(K_{D}\right.$ values of $C s n R$ were respectively 0.032 and $0.040 \mathrm{nM}$ for csnA and csnR operators) (Dubeau et al., 2011).

The role of Rok7B7 in the transcriptional control of aveA1 and $\mathrm{olmRI}$ was further examined using $5^{\prime}$ rapid amplification of CDNA ends (5' RACE) to identify promoter structures of the two genes (Supporting Information Fig. S5). The aveA1 TSS was localized to a $\mathrm{G}$ located $137 \mathrm{nt}$ upstream of the aveA1 translational start codon (TSC) (Fig. 5E), and the olmRI TSS was mapped to a G located $138 \mathrm{nt}$ upstream of the olmRI TSC (Fig. 5E). Rok7B7-binding site $\mathrm{DR}^{\text {ave }}$ on ave $A 1$ promoter region extends from positions -7 to +6 relative to aveA1 TSS and is close to the putative -10 region (Fig. 5E). Binding of repressor close to -10 sequence is common. It is likely that Rok7B7 inhibits aveA1 transcription by hindering recruitment of RNA polymerase. Rok7B7binding site $\mathrm{DR}^{\mathrm{olm}}$ on olmRI promoter region extends from positions -12 to +1 relative to olmRI TSS and overlaps the putative -10 region (Fig. $5 \mathrm{E}$ ). Binding of transcriptional activator to -10 sequence is unusual; however, the binding site of master developmental repressor BldD in the eryBVI promoter region overlaps the -10 sequence, and BldD positively regulates erythromycin production (Chng et al., 2008). Analogously, the SARP (Streptomyces antibiotic regulatory protein)-family regulators, e.g., AfsR (Tanaka et al., 2007), FdmR1 (Chen et al., 2008), and OtcR (Yin et al., 2015), interact with direct repeats close to the -10 regions and presumably activate transcription of target genes by recruiting RNA polymerase to the promoters. The regulatory mechanism of transcriptional activation in the present case remains to be elucidated.

FIG. 4. Rok7B7 directly regulates aveA1 and olmRI.

A. Transcriptional profile of rok7B7 in WT grown in FM-I. Transcription level of rok7B7 on day 1 was defined as 1.

B. Western blotting analysis of Rok7B7 protein level during fermentation process. Rok7B7 temporal expression in strain $\Delta$ rok7B7/rok7B7-3FLAG grown in FM-I was analysed using anti-FLAG mAb. Loading control: Coomassie blue staining of $80 \mu \mathrm{g}$ total protein loaded per lane.

C. Schematic representation of promoter probes for EMSAs.

D. EMSAs of interactions of $\mathrm{His}_{6}-\mathrm{Rok} 7 \mathrm{~B} 7$ with the indicated promoter probes. Negative probe: $h r d B p$. Each lane contained 0.3 nM labelled probe. Concentrations of His ${ }_{6}{ }^{-R o k 7 B 7}$ for probes aveA1p and olmRIp: 250, 500, and $750 \mathrm{nM}$; for other probes: 500 and $750 \mathrm{nM}$; $750 \mathrm{nM} \mathrm{His}_{6}{ }^{-}$ Rok7B7 was used for competition assays (lanes +). Lanes -: EMSAs without His ${ }_{6}$-Rok7B7. Lanes N and S: competition experiments with $\sim 300$-fold excess of unlabelled nonspecific probe $h r d B p(N)$ and specific probe (S). Arrow: free probe. Bracket: Rok7B7-DNA complex.

E. ChIP-qPCR assays of Rok7B7 binding to aveA1p, olmRlp, and aveRp. Anti-FLAG mAb against Rok7B7-3FLAG was used to immunoprecipitate Rok7B7-3FLAG-DNA complexes from 1, 2, 3, 4, 5, and 6 days cultures of $\Delta$ rok7B7/rok7B7-3FLAG treated with formaldehyde. WT strain was used as negative control. Y-axis: relative value of Rok7B7 enrichment on each site, determined by comparison of qPCR cycle number for ChIP sample with that for input DNA.

F. qRT-PCR analysis of aveA1, aveA2, and olmRI in WT and $\Delta$ rok7B7 grown in FM-I. Transcription level was calculated relative to WT level on day 2, defined as 1. Error bars (panels A, E, F): SD from triplicate experiments. Statistical notations (panels E, F): NS, not significant; ${ }^{\star} P<0.05$; ${ }^{\star \star \star} P<0.001$ for comparison with WT ( $t$-test). [Color figure can be viewed at wileyonlinelibrary.com] 
A

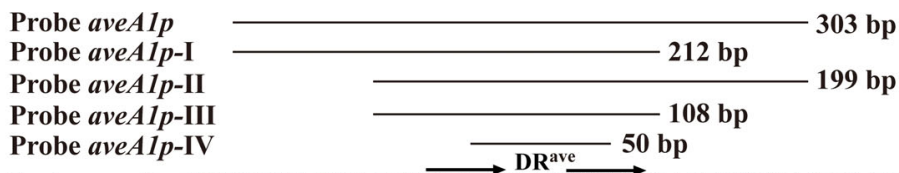

Probe aveAlp-IV $\mathrm{DR}^{\text {ave }} \mathbf{5 0}$ bp

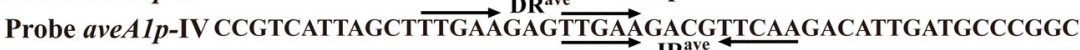
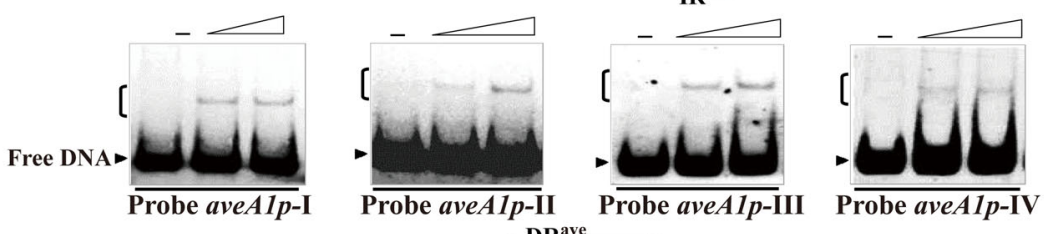

B

Probe aveA1p-IV CCGTCATTAGCTTTGAAGAGTTGAÄGACGTTCAAGACATTGATGCCCGGC Probe 1m CCGTCATTAgCTCCATGgagtTGaAgACGTTCAAGaCATTGATGCCCGGC Probe 2m CCGTCATTAGCTTTGAAGAGCCATGGACGTTCAAGACATTGATGCCCGGC Probe 3m CCGTCATTAGCTTTGAagagtTGAagaCGCCATGGACATTGATGCCCGGC Probe 4m CCGTCATTAGCTCCATGGaGACGCGGACGTTCAAGACATTGATGCCCGGC

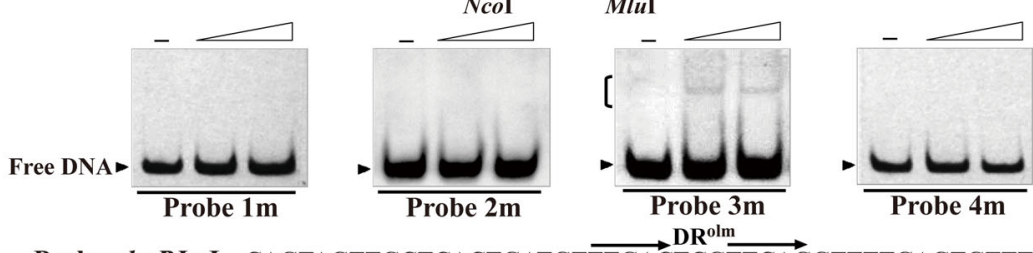

C Probe olmRIp-I CAGTAGTTGCTCACTGATGTTTGACTCGTTCAGGTTTTGAGTGTTTGGAG Probe 5m CAGTAGTTGCTCACTGATGTCCATGTCGACGCGGTTTTGAGTGTTTGGAG

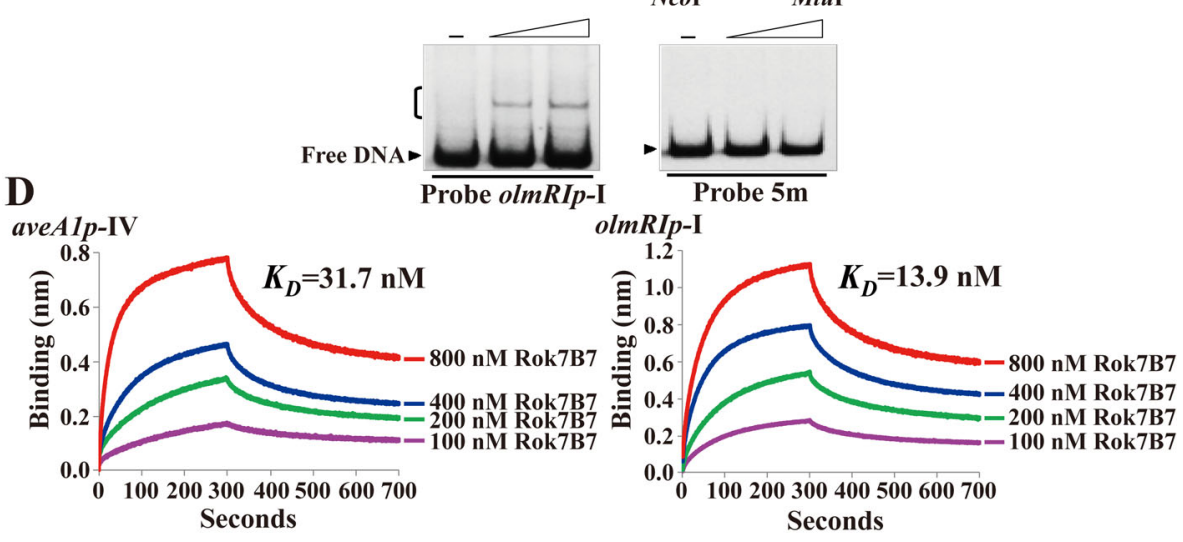

E

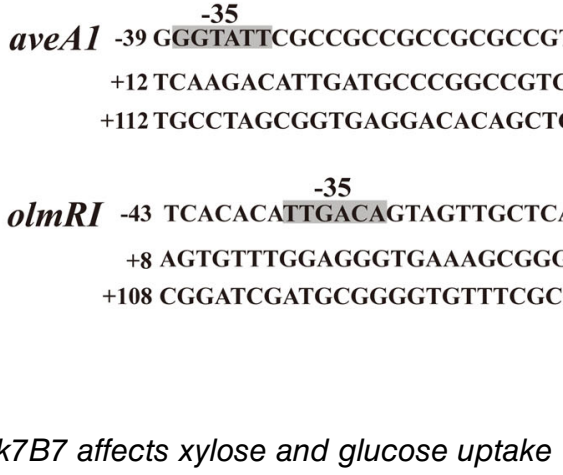

ROK-family regulators are generally associated with sugar metabolism, and S. coelicolor Rok7B7 was shown to affect xylose and glucose uptake (Swiatek et al., 2013). We therefore investigated the role of $S$. avermitilis Rok7B7 in control of sugar uptake. Growth of WT, $\Delta$ rok7B7, and
Fig. 5. Rok7B7-binding sites on promoter regions of aveA1 and olmRI.

A. EMSAs of $\mathrm{His}_{6}-\mathrm{Rok}_{7 \mathrm{~B} 7}$ with probes located within aveA1 promoter region. Relative probe positions and lengths are shown schematically. Straight arrows: inverted or direct repeats. Lanes 2 and 3: 500 and $750 \mathrm{nM} \mathrm{His}{ }_{6}$-Rok7B7.

B. EMSAs using mutated 50-bp probes of aveA1p-IV. Mutations were introduced into inverted or direct repeats of WT probe aveA1p-IV to produce mutated probes $1 \mathrm{~m}, 2 \mathrm{~m}, 3 \mathrm{~m}$, and $4 \mathrm{~m}$. Shading: altered nucleotides.

C. EMSAs using 50-bp WT probe olmRlp-I and its mutated probe $5 \mathrm{~m}$. Direct repeats in probe olmRIp-I were replaced with $\mathrm{NcOl}$ and $\mathrm{Mlul}$ sites to produce mutated probe $5 \mathrm{~m}$.

D. Interaction kinetics of Rok7B7 with probes aveA1p-IV and olmR/p-I determined by BLI analysis. Biotin-labelled DNA probe $(300 \mathrm{nM})$ was loaded on streptavidin sensor and interacted with $\mathrm{His}_{6}$-Rok7B7 at concentrations 100, 200, 400 , and $800 \mathrm{nM}$.

E. Nucleotide sequences of aveA1 and olmRI promoter regions and Rok7B7binding sites. Numbers: distance (nt) from respective TSS. Red bent arrows: TSSs. Shading: presumed -10 and -35 regions. Boxes: TSCs. Underlining: Rok7B7-binding sites. Straight arrows: direct repeats. [Color figure can be viewed at wileyonlinelibrary.com] 
A
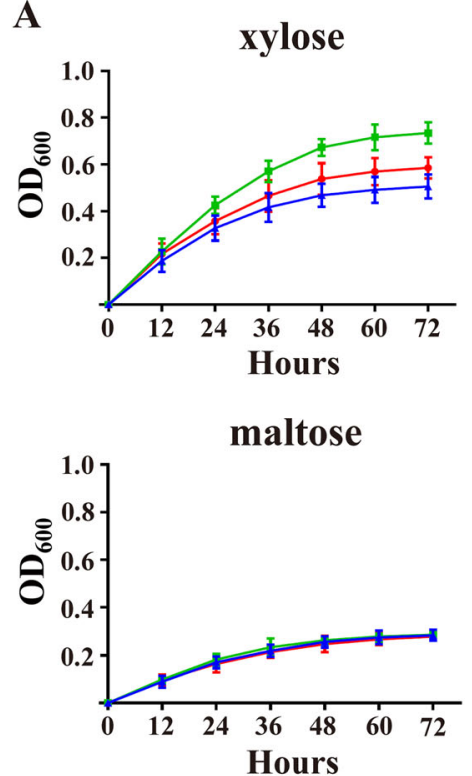

glucose

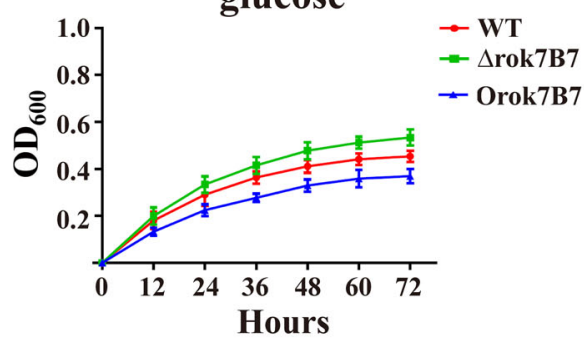

mannitol

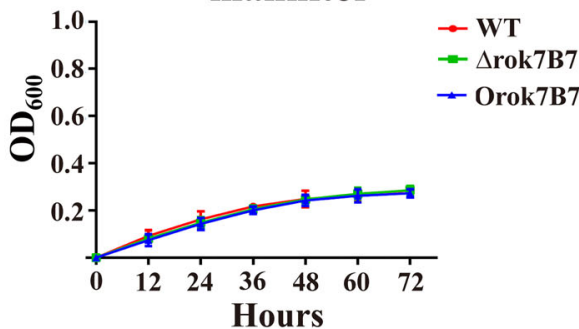

Fig. 6. Effects of Rok7B7 on xylose and glucose uptake.

A. Growth curves of WT, $\triangle$ rok7B7, and Orok7B7 in liquid $\mathrm{MM}$ with various carbon sources (10 $\left.\mathrm{mg} \mathrm{mL}^{-1}\right)$. Biomass is expressed as $\mathrm{OD}_{600}$ (optical density at wavelength $600 \mathrm{~nm}$ ). B. Content of carbon sources remaining in liquid MM after $72 \mathrm{~h}$ culture of the above strains. Error bars: SD from triplicate experiments. NS, not significant; ${ }^{\star \star} P<0.01 ;{ }^{\star \star *} P<0.001$ for comparison with WT (t-test). [Color figure can be viewed at wileyonlinelibrary.com]

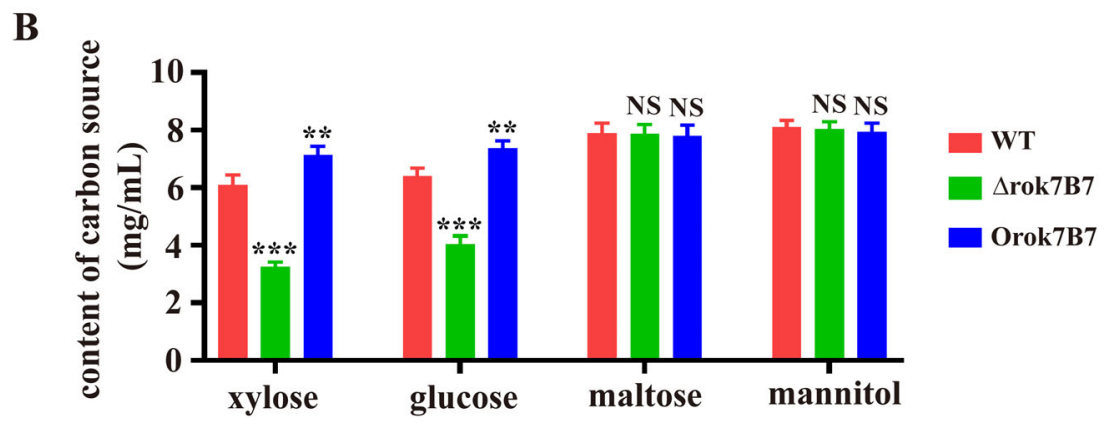

glucose, respectively, after $72 \mathrm{~h}$ culture, while $\Delta$ rok7B7 consumed $67 \%$ of xylose and $59 \%$ of glucose, and Orok7B7 consumed $29 \%$ of xylose and $25 \%$ of glucose (Fig. 6B). With maltose or mannitol as carbon source, growth rate and biomass yield did not differ appreciably among the three strains (Fig. 6A). Consumption of maltose and mannitol by the three strains was similar, i.e., $\sim 20 \%$ for maltose and $\sim 19 \%$ for mannitol (Fig. $6 B$ ). These findings indicate that Rok7B7 represses xylose and glucose uptake in S. avermitilis.

\section{Rok7B7 directly represses the xylFGH operon}

The xylose transport operon $x y / F G H$ is adjacent to rok7B7, and 5-bp direct repeats (TTTACTTCTTGAC; termed $D R^{x y l}$ ) similar to those in sequence $D R^{\text {ave }}$ are present in promoter region of $x y I F$. To test if Rok7B7 regulates the $x y I F G H$ operon, EMSAs were performed using probe $x y I F p$ that contains the $x y I F G H$ promoter region. Indeed, $\mathrm{His}_{6}-\mathrm{Rok} 7 \mathrm{~B} 7$ was able to interact with probe $x y l F p$, whereas $\mathrm{His}_{6}$-Rok7B7 did not auto-regulate its own transcription (probe rok7B7p) (Fig. 7A). Direct binding of Rok7B7 to $x y / F p$ but not to rok7B7p was confirmed in vivo by ChIP-qPCR assays (Fig. 7B).

The role of sequence $D^{x y l}$ in Rok7B7 binding was examined by performing site-directed mutagenesis of 50-bp WT probe $x y I F p-I$ (containing intact $D^{x y l}$ ) on the direct repeats to generate mutated probe $6 \mathrm{~m}$, which lacked direct repeats (Fig. 7C). Binding of Rok7B7 to WT probe $x y I F p-I$ and to $6 \mathrm{~m}$ was evaluated by EMSAs. $\mathrm{His}_{6}{ }^{-}$ Rok7B7 bound to $x y l F p$-l but not to $6 \mathrm{~m}$ (Fig. 7C), indicating that the direct repeats in $D^{x y l}$ are essential for Rok7B7 binding.

$\mathrm{BLI}$ assays revealed a $K_{D}$ of Rok7B7/ $x y \mid F p-I$ interaction of $47.5 \mathrm{nM}$ (Fig. 7D), which is lower than for aveA1pIV $\left(K_{D}=31.7 \mathrm{nM}\right)$ or olmRIp-I $\left(K_{D}=13.9 \mathrm{nM}\right)$. qRT-PCR analysis showed that transcript levels of $x y / F, x y I G$ and $x y l H$ were higher for $\triangle$ rok7B7 than for WT grown in FM-I (Fig. 7E), and thus that Rok7B7 represses the operon.

Most Streptomyces strains contain another $x y l$ gene locus, $x y I A B R$, involved in xylose uptake. XylA (xylose isomerase) catalyses isomerization of xylose to xylulose, and XylB (xylulose kinase) mediates phosphorylation of xylulose to xylulose-5-phosphate. XyIR acts as a 
A

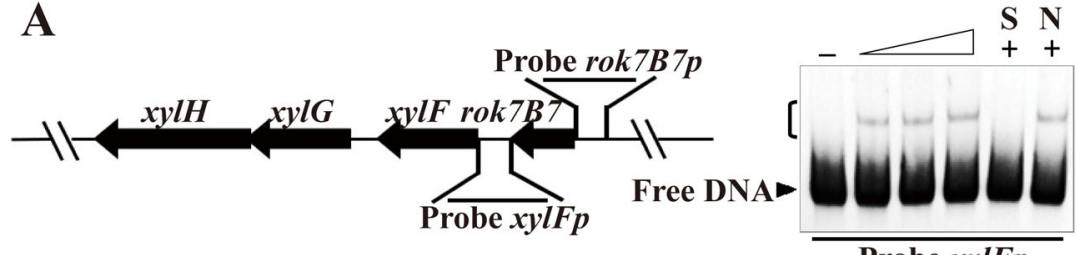

Probe $x y l F p$

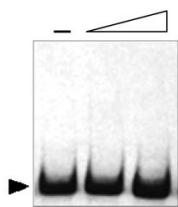

B

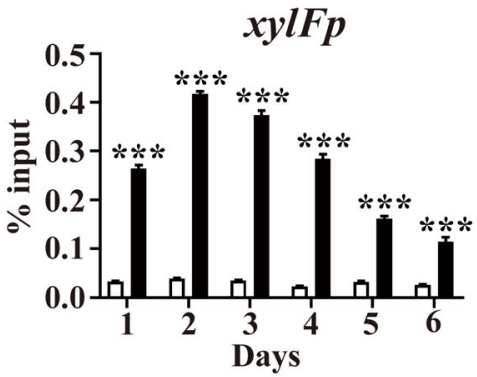

$\operatorname{rok} 7 \mathrm{~B} 7 \mathrm{p}$

Probe $\operatorname{rok} 7 \mathrm{~B} 7 \mathrm{p}$

C

Probe $x y l F p$-I ATCTCGTTAAGTATTTACTTCTTGACGTCGCACGTGTGGCCGAGTTGACT

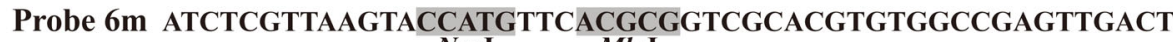
Ncol MluI
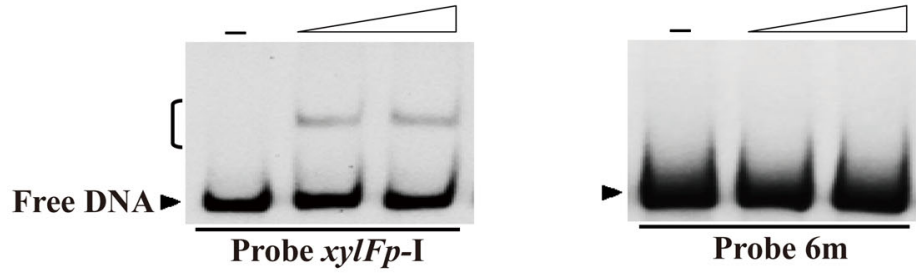

Probe 6m

D

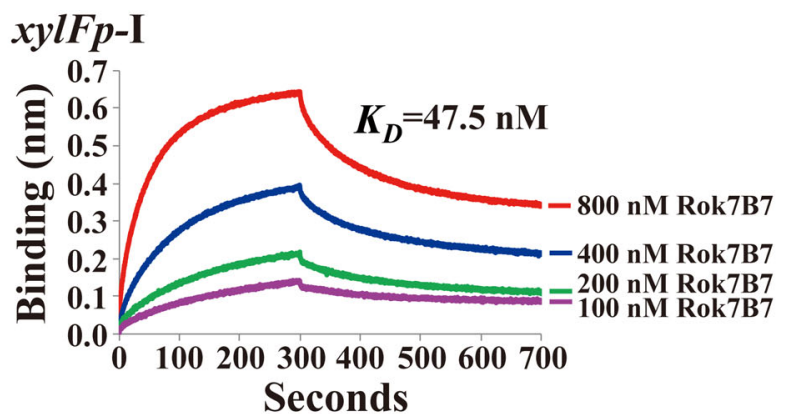

$\mathbf{E}$
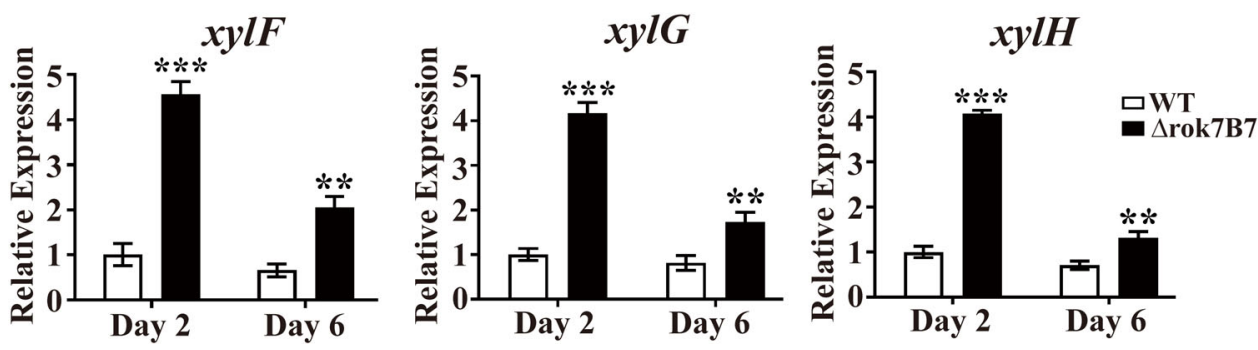

Fig. 7. Rok7B7 directly regulates $x y I F G H$ operon.

A. EMSAs of His ${ }_{6}$-Rok7B7 using probes $x y I F p$ and rok7B7p. His ${ }_{6}$-Rok7B7 concentrations for probe $x y l F p$ : 250, 500, and $750 \mathrm{nM}$; for probe rok7B7p: 500 and $750 \mathrm{nM}$. Lane notations $(-,+, \mathrm{N}, \mathrm{S})$ as in Fig. 4D.

B. ChIP-qPCR assays of Rok7B7 binding to $x y I F p$ and rok7B7p. Notations as in Fig. 4E.

C. EMSAs using 50-bp WT probe $x y I F p-I$ and its mutated probe $6 \mathrm{~m}$. Direct repeats in probe $x y / F p-I$ were replaced with $N c o l$ and $M l u l$ sites to produce mutated probe $6 \mathrm{~m}$. Lanes 2 and 3 contained 500 and $750 \mathrm{nM} \mathrm{His} 6$-Rok7B7.

D. BLI analysis of $\mathrm{His}_{6}-\mathrm{Rok}_{7 \mathrm{~B} 7}$ binding to probe $x y I F p-I$.

E. qRT-PCR analysis of $x y / F, x y / G$, and $x y l H$ in WT and $\Delta$ rok7B7 grown in FM-I. Panels B, E: error bars: SD from triplicate experiments; NS, not significant; ${ }^{* *} P<0.01 ;{ }^{* * *} P<0.001$ for comparison with WT (t-test). [Color figure can be viewed at wileyonlinelibrary.com] 
repressor of $x y \mid A$ and $x y I B$ (Swiatek et al., 2013; Noguchi et al., 2018). Gene numbers for $x y I A B R$ in $S$. avermitilis are sav_7182 (xylA), sav_7181 (xylB1), and sav_7180 $(x y / R) . x y / B 1$ is transcribed divergently from $x y / A$, and convergently with $x y I R$ (Supporting Information Fig. S6A). However, binding of Rok7B7 to probe $x y / R p$ or $x y\left|A \_x y\right| B 1$ (containing bidirectional promoters) was not observed (Supporting Information Fig. S6B).

\section{Impact of rok7B7 deletion on xylose and glucose uptake}

To investigate the effects of xylose and glucose on expression of $x y I F G H$, transcription of $x y I F$ was analysed by qRT-PCR using RNAs isolated from WT or $\triangle$ rok7B7 grown in liquid MM with xylose, glucose or xylose/glucose mixture as carbon sources. In WT, $x y / F$ transcription was enhanced by xylose within $12 \mathrm{~h}$, but was strongly repressed in the presence of glucose and xylose/glucose (Fig. 8A), indicating that the $x y / F G H$ operon is subject to CCR. In $\triangle$ rok7B7, xylF transcription was greatly increased under all conditions (Fig. 8A), consistent with repression of $x y / F G H$ by Rok7B7. Notably, glucose had no effect on $x y I F$ transcription in $\triangle$ rok7B7, indicating that the repressing effect of glucose on $x y I F G H$ is mediated by Rok7B7. Xylose induced $x y / F$ transcription in $\Delta$ rok7B7, suggesting that $x y / F G H$ operon is induced by xylose in both Rok7B7-dependent and -independent manners.

In a study of co-uptake of xylose and glucose by WT and $\triangle$ rok7B7 in liquid MM cultures containing xylose and glucose, WT consumed $33 \%$ of glucose but only $4 \%$ of xylose during $72 \mathrm{~h}$ culture (Fig. 8B). In contrast, $\triangle$ rok7B7 consumed glucose and xylose simultaneously, and eventually consumed $25 \%$ of glucose and $38 \%$ of xylose. Total sugars consumption was $63 \%$ for $\Delta$ rok7B7 and $37 \%$ for WT; i.e., co-uptake efficiency of xylose and glucose was greatly improved by rok7B7 deletion.

\section{Xylose and glucose act as ligands of Rok7B7}

To test whether xylose and glucose can act as ligands, EMSAs were performed to check the effect of the sugars on the affinity of Rok7B7 for $x y / F p$. DNA-binding ability of Rok7B7 was impaired in the presence of xylose, but enhanced in the presence of glucose in a concentrationdependent manner (Fig. 8C). These findings were supported by BLI assays using probe xylFp-I (Fig. 8D), which showed that xylose and glucose serve as Rok7B7 ligands, but play opposite roles in modulating DNAbinding ability of Rok7B7. These findings, in combination with those from transcription analysis (Figs. 7E and 8A), allowed us to formulate a model of control of xylose uptake by Rok7B7 in response to xylose and glucose. Xylose acts as an inducer and, in the absence of glucose, releases Rok7B7 from $x y / F p$, resulting in increased $x y I F G H$ expression for xylose uptake. In contrast, in the presence of a preferred carbon source as glucose, the affinity of Rok7B7 for its target site is enhanced.

To investigate whether the Rok7B7 orthologs from model strains $S$. coelicolor and $S$. venezuelae had similar binding affinities, the proteins were expressed as $\mathrm{His}_{6}{ }^{-}$ tagged proteins in E. coli and purified, and EMSAs were performed on the promoter region of $x y / F s c o$ and $x y I F s v e n$. This showed that also the Rok7B7 proteins of $S$. coelicolor and $S$. venezuelae bound to their respective xyIFGH promoters (Supporting Information Fig. S7). DNA-binding affinity of the two proteins was inhibited by xylose, but enhanced by glucose (Supporting Information Fig. S7). These findings suggest wide conservation of the cis-trans relationships for Rok7B7 with the $x y / F G H$ operon and its control by glucose CCR in Streptomyces species.

\section{Prediction and verification of new Rok7B7 target genes}

Understanding of the broader roles of Rok7B7 in S. avermitilis requires identification of additional Rok7B7 target genes. Analysis of the 13-bp direct repeat sequences in the three Rok7B7-binding promoter regions mentioned above (aveA1p, olmR/p, xylFp) using WebLogo programme (http://weblogo.berkeley.edu) revealed a consensus sequence $5^{\prime}$-TTKAMKHSTTSAV- $3^{\prime}$ $(K=T / G ; M=A / C ; H=A / C / T ; S=C / G ; V=A / C / G)$ (Fig. 9A). Scanning of the $S$. avermitilis genome by PREDetector (Hiard et al., 2007) with the 13-bp consensus Rok7B7-binding sequence identified 275 putative Rok7B7 target genes (cut-off score $\geq 7$ ) (Supporting Information Table S1). Of these, 82 were unknown or unclassified, and the remaining 193 were assigned to 17 groups on the basis of biological function as defined by the KEGG pathway database for S. avermitilis. Accuracy of the bioinformatic prediction was tested by selecting 23 well-annotated putative targets involved in primary metabolism, secondary metabolism, or development for EMSA confirmation.

Interestingly, one of the putative Rok7B7 target genes, sav_2657, encodes glucose transporter GlcP (van Wezel et al., 2005). While the gene was annotated as araE (for a putative L-arabinose permease) by the Genome Project of S. avermitilis (http://avermitilis.Is.kitasato-u.ac.jp), the gene product is identical to glucose permease GlcP in S. coelicolor (van Wezel et al., 2005). Rok7B7 bound well to probe sav_2657p (Fig. 9B). sav_2657 transcription was significantly higher after 2 and 6 days for $\Delta$ rok7B7 grown in FM-I than for WT, indicating that Rok7B7 acts as a repressor of this gene (Fig. 9C). Analysis of WT and $\triangle$ rok7B7 grown in liquid MM containing 
A
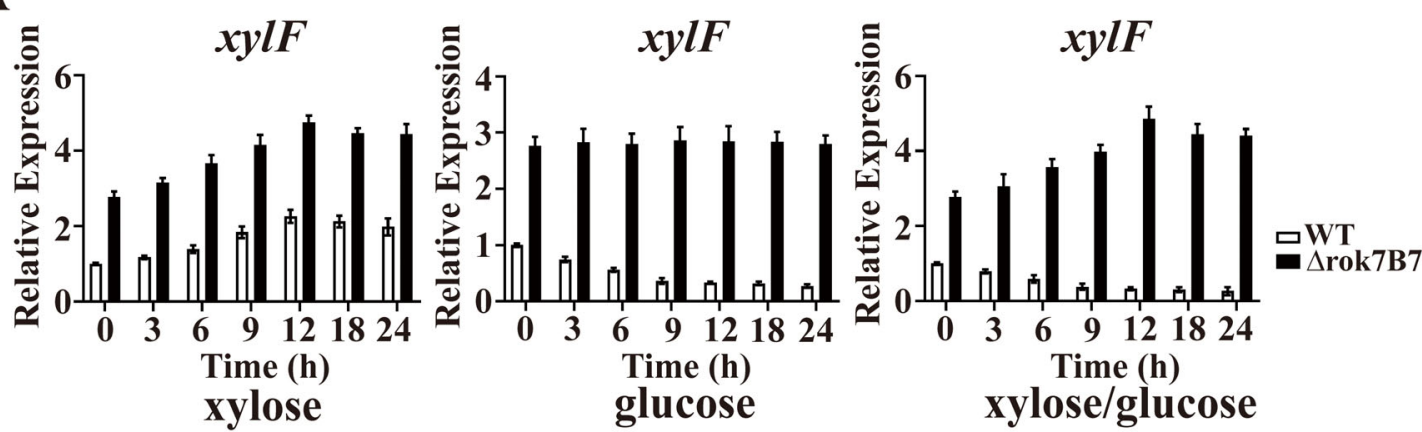

B

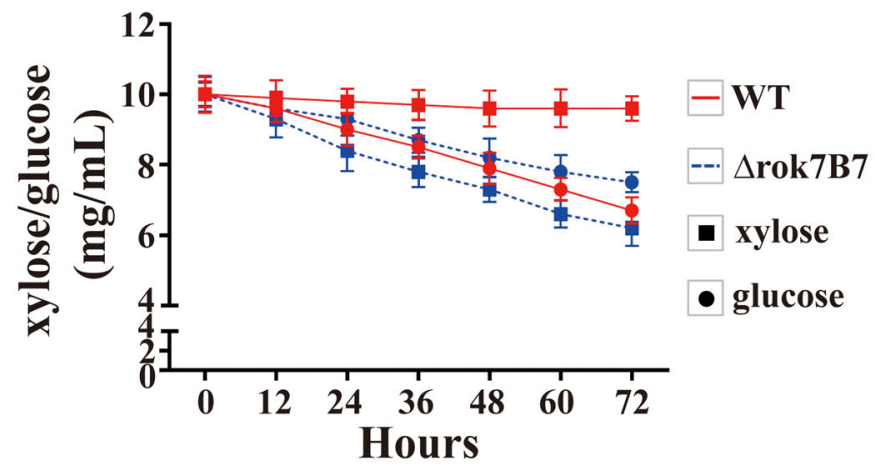

C

D
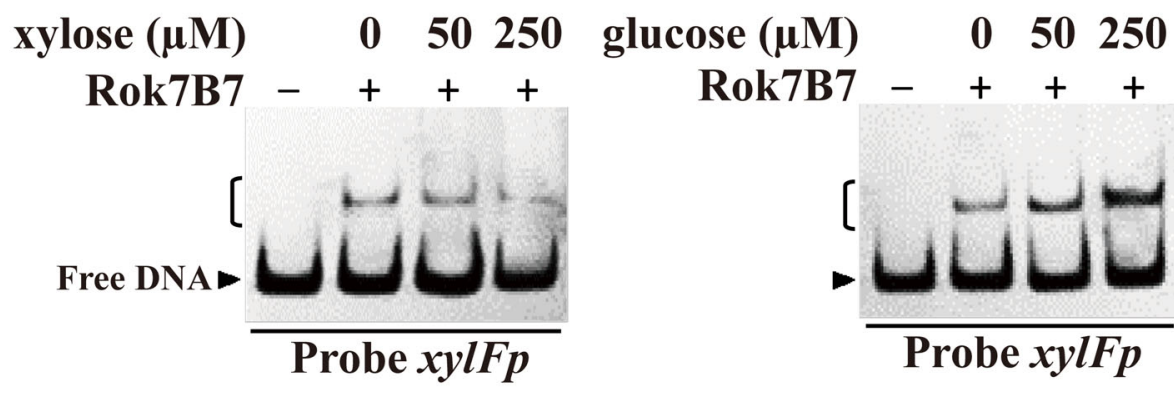

$x y l F p-I$
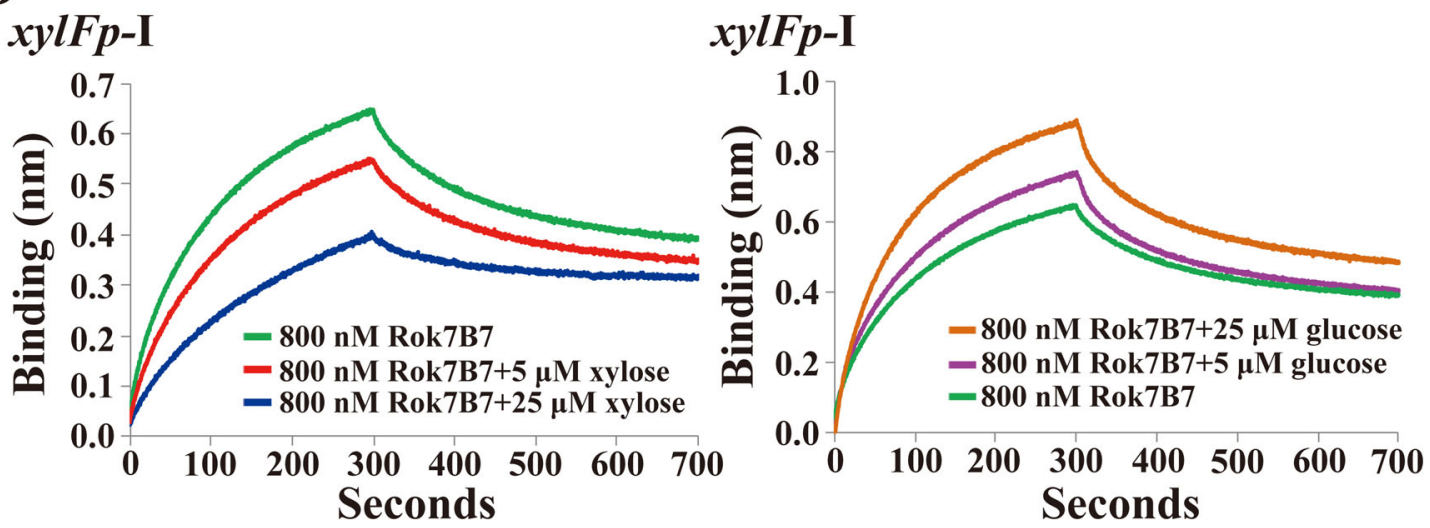

Fig. 8. Effects of xylose and glucose on DNA-binding activity of Rok7B7 to $x y I F p$.

A. QRT-PCR analysis of $x y I F$ in WT and $\Delta$ rok7B7 grown in MM containing xylose, glucose, or xylose/glucose combination. Cells were grown in MM containing mannitol $\left(2 \mathrm{mg} \mathrm{mL}^{-1}\right)$ for $60 \mathrm{~h}$, followed by addition of various sugars (each $\left.10 \mathrm{mg} \mathrm{mL}^{-1}\right)$. $x y / F$ transcription level in WT before sugar addition $(0 \mathrm{~h})$ was defined as 1 .

B. Consumption of glucose and xylose by WT and $\Delta$ rok7B7 grown in MM containing xylose/glucose mixture $\left(10 \mathrm{mg} \mathrm{mL}^{-1}\right)$. Error bars (panels A,

B): SD from triplicate experiments.

C. EMSAs of $\mathrm{His}_{6}-\mathrm{Rok7B7}(500 \mathrm{nM})$ with xylose and glucose at indicated concentrations.

D. BLI analysis of $\mathrm{His}_{6}-$ Rok7B7 $(800 \mathrm{nM})$ with xylose and glucose. [Color figure can be viewed at wileyonlinelibrary.com] 
A
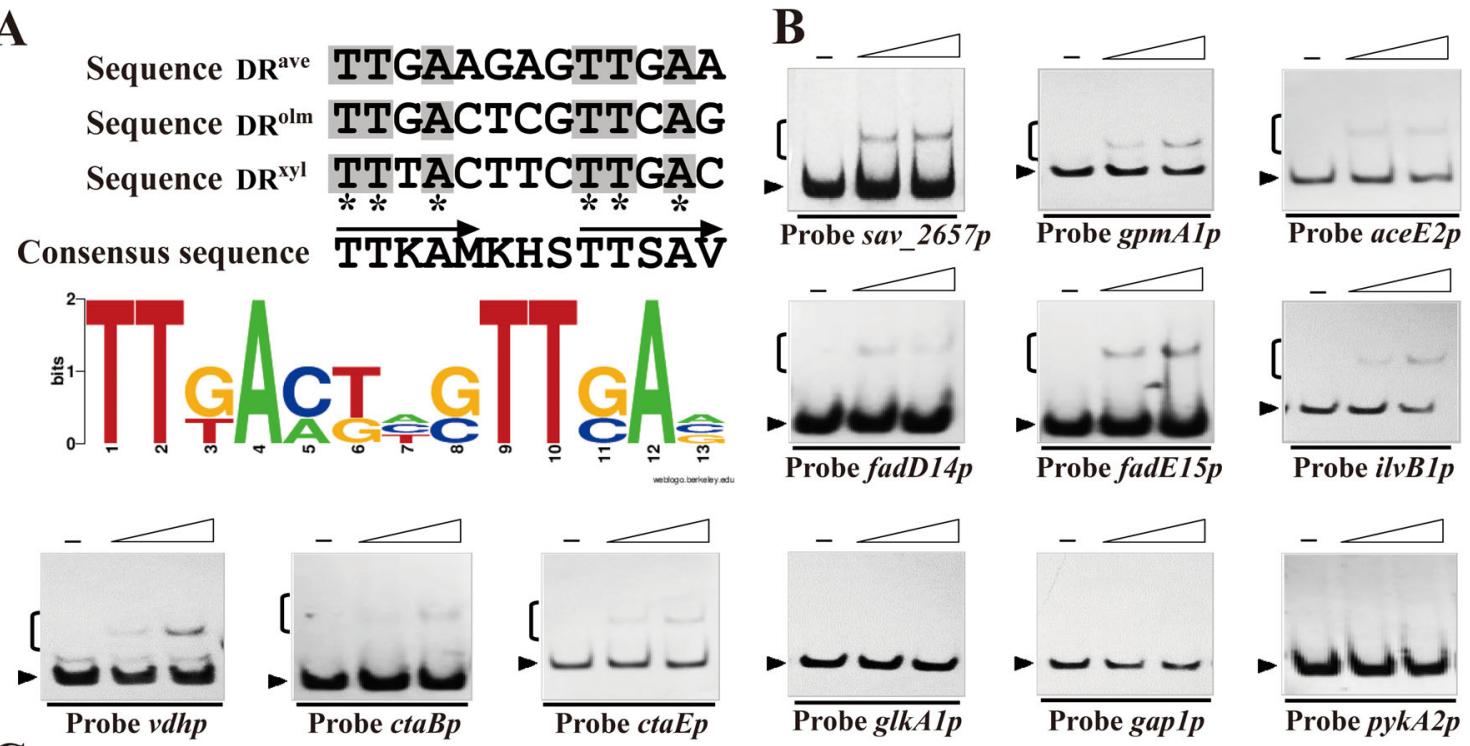

C
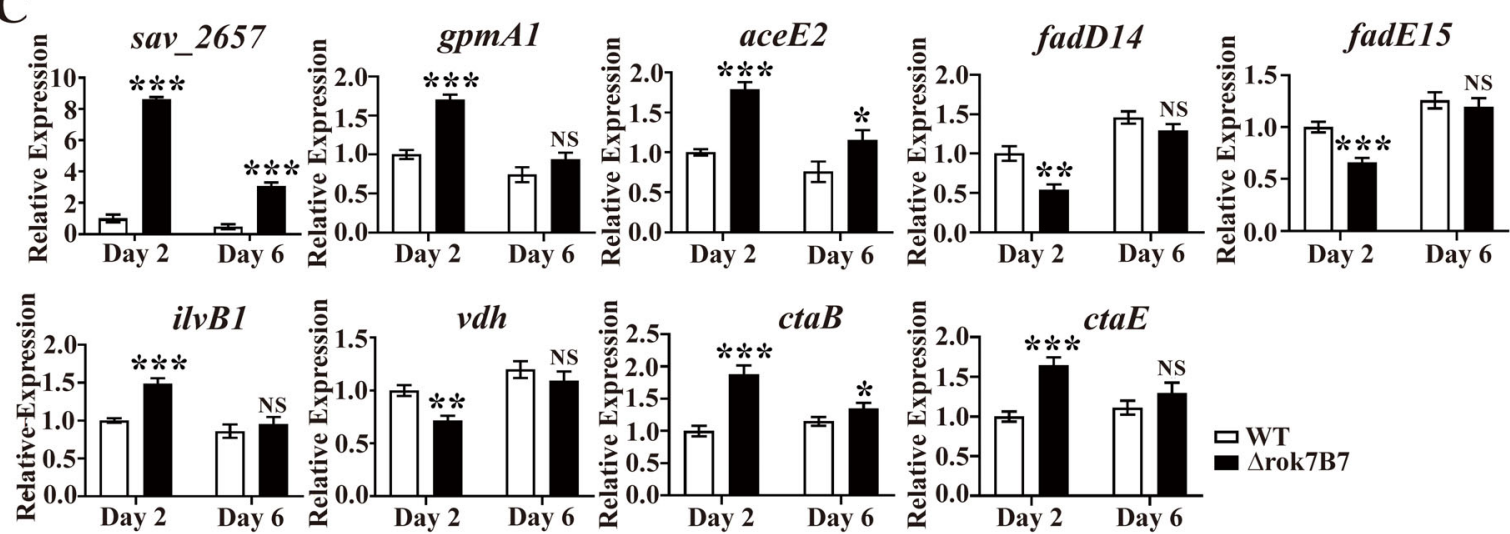

D
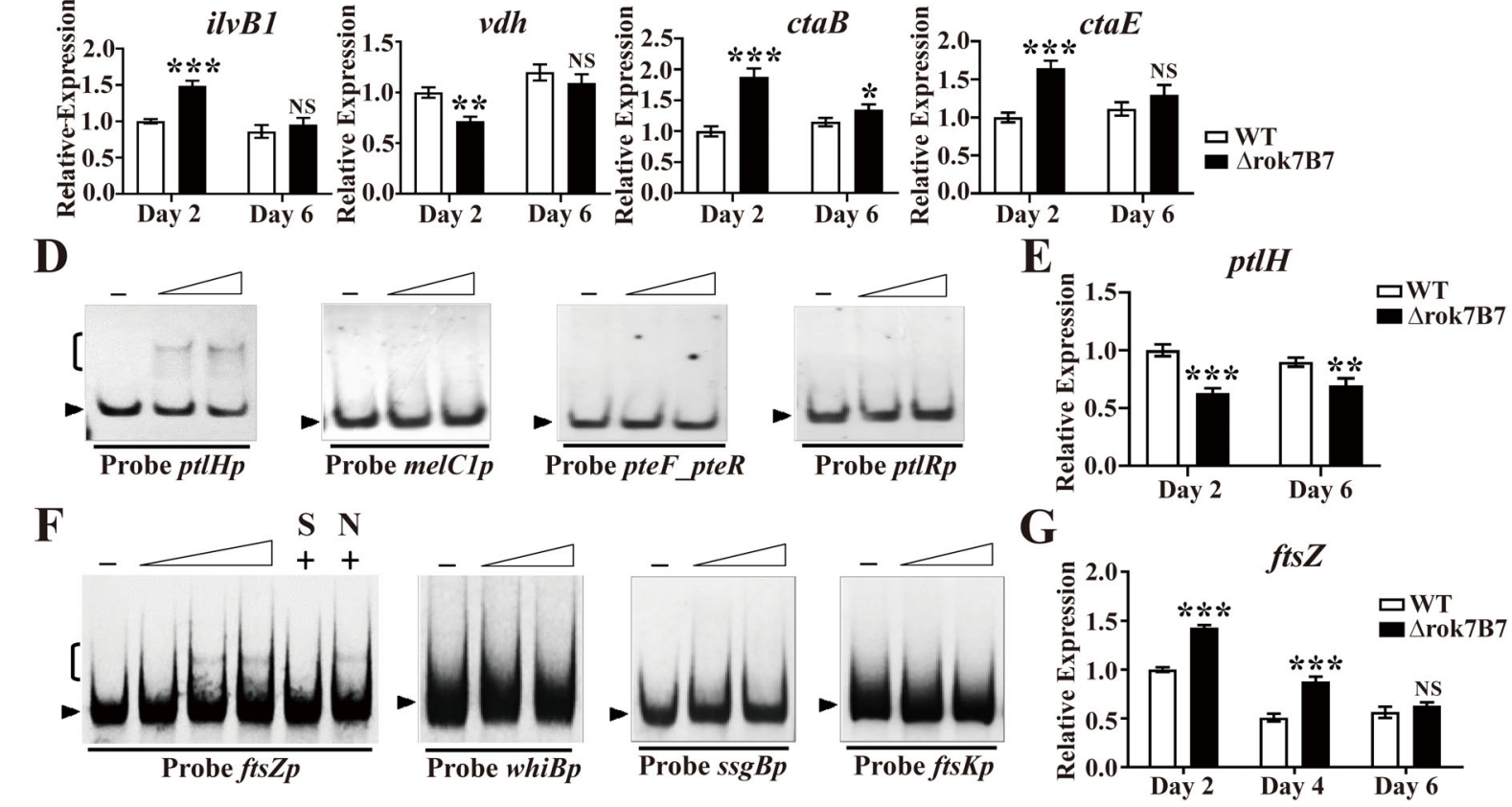

G

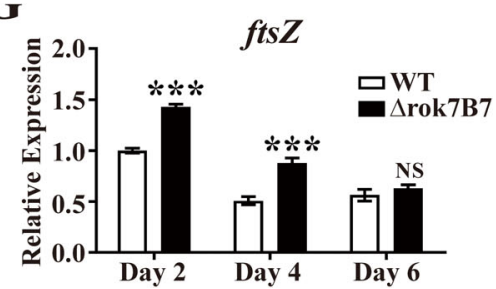

Fig. 9. Identification of new Rok7B7 targets.

A. Analysis of consensus Rok7B7-binding sequence by WebLogo programme. Arrows: conserved 5-bp direct repeats. Asterisks: consensus bases.

B. EMSAs of $\mathrm{His}_{6}$-Rok7B7 with promoter regions of 12 predicted target genes associated with primary metabolism. Lanes 2 and 3 contained 500 and $750 \mathrm{nM} \mathrm{His} 6$-Rok7B7.

C. qRT-PCR analysis of sav_2657, gpmA1, aceE2, fadD14, fadE15, ilvB1, vdh, ctaB, and ctaE in WT and $\Delta$ rok7B7 grown in FM-I.

D. EMSAs of $\mathrm{His}_{6}$-Rok7B7 with promoter regions of five predicted target genes associated with secondary metabolism.

E. qRT-PCR analysis of $p t / H$ in WT and $\Delta$ rok7B7 grown in FM-I.

F. EMSAs of $\mathrm{His}_{6}$-Rok7B7 with four predicted target promoters associated with development. His ${ }_{6}$-Rok7B7 concentrations for probe ftsZp: 250, 500, and $750 \mathrm{nM}$; for other promoter probes: 500 and $750 \mathrm{nM}$.

G. qRT-PCR analysis of ftsZ in WT and $\triangle$ rok7B7 grown on YMS plates. Panels C, E, G: error bars: SD from triplicate experiments; NS, not significant; ${ }^{*} P<0.05 ;{ }^{\star \star} P<0.01$; ${ }^{* \star \star} P<0.001$ for comparison with WT (t-test). [Color figure can be viewed at wileyonlinelibrary.com] 
arabinose, glucose, or arabinose/glucose mixture showed that sav_2657 transcription was strongly induced by glucose, but unaffected by arabinose (Supporting Information Fig. S8). sav_2657 transcription level was also notably increased by rok7B7 deletion in each of the above three culture conditions. These findings suggest that Rok7B7 affects glucose uptake by directly repressing the gene sav_2657 for the glucose permease GlcP.

EMSAs were performed on 11 additional putative Rok7B7 target genes associated with primary metabolism: glkA1, gap1, gpmA1, pykA2, aceE2, fadD14, fadE15, ilvB1, vdh, ctaB, and ctaE (Supporting Information Table S1). Rok7B7 bound directly to promoter regions of gpmA1, aceE2, fadD14, fadE15, ilvB1, vdh, $c t a B$, and $c t a E$, but not to those of glkA1, gap1, or pykA2 (Fig. 9B). qRT-PCR analysis revealed that transcription levels of gpmA1, aceE2, ilvB1, ctaB, and $c t a E$ were higher in $\triangle$ rok7B7 than in WT, whereas those of fadD14, fadE15, and $v d h$ were lower in $\Delta$ rok7B7 (Fig. 9C). These findings demonstrate that Rok7B7 plays a pleiotropic role and displays dual repressor/activator function in primary metabolism.

Several putative Rok7B7 targets associated with secondary metabolism were also selected for EMSA evaluation: $m e / C 1$ involved in melanin biosynthesis; $p t e F$ and $p t e R$ in filipin biosynthesis (Vicente et al., 2014); ptIR and $p t / H$ in biosynthesis of unknown terpene metabolite (Supporting Information Table S1). Rok7B7 bound only to probe $p t / H p$, but not to melC1p, pteF-pteR (bidirectional), or pt/Rp (Fig. 9D). ptlH transcription level was reduced in $\triangle$ rok7B7 (Fig. 9E), indicating that Rok7B7 acts as an activator of this newly identified target related to secondary metabolism.

In addition, several key genes involved in the onset of sporulation-specific cell division were included in the list of putative Rok7B7 targets, namely whiB (sav_5042) for the sporulatin protein WhiB (Bush et al., 2016), ftsZ (sav_6124) for the tubulin ortholog FtsZ that forms the cell division scaffold (Willemse et al., 2011; Schwedock et al., 1997), ssgB (sav_4604) for SsgB that recruits FtsZ to septum sites and thus activates sporulation-specific cell division (Willemse et al., 2011; Keijser et al., 2003), and ftsK (sav_2510) for FtsK that is required for chromosome segregation during cell division (Dedrick et al., 2009). Rok7B7 bound only to ftsZp, while it failed to bind to the other targets (Fig. 9F). qRT-PCR analysis of $f t s Z$ transcription level using RNAs extracted from WT and $\triangle$ rok7B7 grown on YMS plates for 2 (aerial growth), 4 (middle stage of sporulation), or 6 days (spore maturation) showed that the level was upregulated in $\triangle$ rok7B7 on days 2 and 4 (Fig. 9G), consistent with the earlier developmental phenotype of $\triangle$ rok7B7. These findings indicate that Rok7B7 negatively regulates development by directly repressing $\mathrm{ftsZ}$.

\section{Discussion}

ROK-family regulator Rok7B7 was shown to play a functional role in S. coelicolor (Swiatek et al., 2013); however, until now little was known regarding its regulatory targets. We examined the molecular mechanism underlying Rok7B7 function in S. avermitilis and demonstrated that it functions in this species as an essential global regulator of development, antibiotic production, carbon uptake, and other primary metabolic processes through control of related target genes. Our findings provide an excellent basis for future studies of ROK-family regulators in Streptomyces species, and for construction of antibioticoverproducing strains. We also revealed that Rok7B7 and its orthologs participate in glucose-mediated CCR of xylose uptake through direct regulation of xylose transport operon $x y I F G H$, and DNA-binding activity of Rok7B7 on $x y I F p$ is modulated by glucose and xylose. Interestingly, several previous studies have shown that Streptomyces CCR is mediated through specific transcriptional regulators (Walter and Schrempf, 1996; $\mathrm{Ni}$ and Westpheling, 1997; Nguyen et al., 1997; Hindle and Smith, 1994; van Wezel et al., 1997), e.g., amylase and chitinase genes in S. lividans are regulated by a Lacl/ GalR-family regulator, Reg 1, which is involved in glucose repression (Nguyen et al., 1997); GyIR (Hindle and Smith, 1994) and MalR (van Wezel et al., 1997), which control glycerol and maltose uptake in $S$. coelicolor, respectively, are required for both substrate induction and CCR. However, the detailed relationship of these regulators and Rok7B7 with GlkA is still unclear and requires further investigations.

Rok7B7 plays a differential role in regulation of avermectin and oligomycin A production, which are mediated respectively by structural genes and CSR gene. It also regulates the expression of the BGC directing the biosynthesis of an unknown terpene metabolite via activation of the expression of the structural gene $\mathrm{pt} / \mathrm{H}$. These findings demonstrate the pleiotropic and diverse role of Rok7B7 in the regulation of the expression of BGC. In many cases, pleiotropic/global regulators directly control CSR genes involved in antibiotic biosynthesis. However, there is increasing evidence that certain regulators, including S. roseosporus AtrA (Mao et al., 2015), S. chattanoogensis WhiG (Liu et al., 2015), S. coelicolor AfsQ1 (Chen et al., 2016), S. avermitilis SAV_742 (Sun et al., 2016), and Rok7B7 (present study) directly control specific antibiotic structural genes. Such direct regulatory mechanism of antibiotic biosynthesis may be universal in streptomycetes and facilitate rapid, precise regulation of antibiotic biosynthesis in response to various signals.

Concerning the control of xylose uptake, Rok7B7 acts as a direct repressor of $x y I F G H$ but not of another $x y l$ gene locus, $x y \mid A B R$, consistent with the observation that 
rok7B7 deletion in S. coelicolor had no effect on $x y \mid A B R$ expression (Swiatek et al., 2013). XyIR repressed xylA and $x y / B$ (Swiatek et al., 2013; Noguchi et al., 2018), and addition of xylose derepressed $x y / A$ expression in S. avermitilis (Noguchi et al., 2018), but the relationship between XyIR and $x y I F G H$ was not previously investigated. In the present study, the expression of the $x y I F G H$ operon completely escaped glucose repression in $\Delta$ rok7B7, but xylose still induced transcription of these genes, suggesting that $x y l F G H$ is controlled by other regulator(s). XyIR is the most likely candidate, thus, its regulatory role on $x y I F G H$ remains to be more fully elucidated. In regard to glucose uptake, $S$. coelicolor contains two GlcP-encoding genes ( $g / c P 1, g / c P 2$ ), and g/cP1 plays a major role in glucose transport (van Wezel et al., 2005). In S. avermitilis, sav_2657 is the only gene homologous to $g / c P 1$ and $g / c P 2$. Expression of sav_2657, as a Rok7B7 target, was strongly induced by glucose, but unaffected by arabinose, and sav_2657 transcription level was strongly upregulated in $\triangle$ rok7B7, consistent with the enhanced glucose uptake in this mutant. Thus, sav_2657 evidently encodes a glucose permease involved in glucose transport, rather than an arabinose permease as annotated in the Genome Project of S. avermitilis.

Glucose and xylose are the major sugar components of lignocellulose, which is present in plant cell walls and has the potential to be developed as a renewable resource for microbial production of biofuels and other value-added bioproducts (Liguori et al., 2016). Streptomyces species produce enzymes used for lignocellulosic biomass hydrolysis (Pennacchio et al., 2018) and therefore can potentially be engineered to produce antibiotics or other useful products from lignocellulose hydrolysates. Efficient conversion of lignocellulosic materials requires simultaneous uptake of the two sugars. A common problem is that the presence of glucose inhibits xylose uptake by CCR. rok7B7 deletion in $S$. avermitilis and S. coelicolor (Swiatek et al., 2013) enhanced both xylose and glucose uptake, and xylose uptake was not inhibited by glucose in $\triangle$ rok7B7 mutant of $S$. avermitilis. rok7B7 orthologous genes are widespread among Streptomyces species, and rok7B7 deletion strategy therefore has great potential to improve co-uptake efficiency of glucose and xylose derived from lignocellulosic biomass and efficiency of fermentation process in streptomycetes.

Besides sav_2657, we identified eight new Rok7B7 targets involved in primary metabolism; i.e., genes involved in glycolysis (gpmA1, aceE2), fatty acid degradation (fadD14, fadE15), valine and isoleucine biosynthesis (ilvB1), valine degradation ( $v d h)$, and oxidative phosphorylation (ctaB, ctaE). These genes are closely associated with antibiotic biosynthesis. Glycolysis yields energy and acetyl-CoA, which become available for avermectin and oligomycin $A$ biosynthesis. Fatty acid degradation provides acetyl-CoA precursor for biosynthesis of these two antibiotics. Starter units for avermectin biosynthesis are derived from valine and isoleucine (Ikeda and Omura, 1997). Thus, ilvB1 and $v d h$ are involved in providing starter units for avermectin biosynthesis. Oxidative phosphorylation is the main energy source for aerobic Streptomyces. Altered expression of $c t a B$ and $c t a E$ may thus affect energy supply for antibiotic biosynthesis. Rok7B7 represses gpmA1, aceE2, ilvB1, ctaB, and ctaE, but activates fadD14, fadE15, and $v d h$. Yield data obtained for avermectins and oligomycin A in $\triangle$ rok7B7 therefore reflect a combined effect of altered expression of these primary metabolic genes. Avermectins and oligomycin A require common extender units for synthesis of polyketide chain (Omura et al., 2001); increase or decrease of antibiotic yields in $\Delta$ rok7B7 may therefore be due in part to precursor competition between these two biosynthetic pathways.

We observed a negative regulatory role of Rok7B7 in $S$. avermitilis development, and identified the central cell division gene fts $Z$ as a Rok7B7 target. During sporulation, FtsZ is recruited to the septum site by SsgB to form a ladder of 50-100 $Z$ rings in each sporogenic hypha to direct synthesis of sporulation septa (Willemse et al., 2011). In the mutant $\Delta$ rok7B7, enhanced fts $Z$ expression contributed to earlier spore formation. However, possible contributions by other development-related Rok7B7 target genes to $\triangle$ rok7B7 phenotype cannot be ruled out; further studies are needed to address this point.

A proposed schematic model of the Rok7B7-mediated regulatory network involved in primary metabolism (including xylose and glucose uptake), secondary metabolism, and development in S. avermitilis is shown in Fig. 10. The coordinated roles of Rok7B7 in these essential physiological processes are achieved through its regulatory effects on target genes. The promoter regions of several putative targets (glkA1, gap1, pykA2, melC1, pteF, pteR, ptIR, whiB, ssgB, $f t s K$ ) listed in the Supporting Information Table S1 were not bound by Rok7B7 in vitro. While we cannot explain this yet, it is tempting to suggest the presence of different classes of binding sites, as was previously observed for DasR (Swiatek-Polatynska et al., 2015). Another possibility is that such binding requires a specific ligand or an additional protein that contributes to its binding. The complex roles of Rok7B7 in streptomycetes will be progressively elucidated as we identify additional target genes and molecular processes.

\section{Experimental procedures}

Strains, plasmids, and growth conditions

Strains and plasmids used or constructed in this study are summarized in the Supporting Information Table S2, 


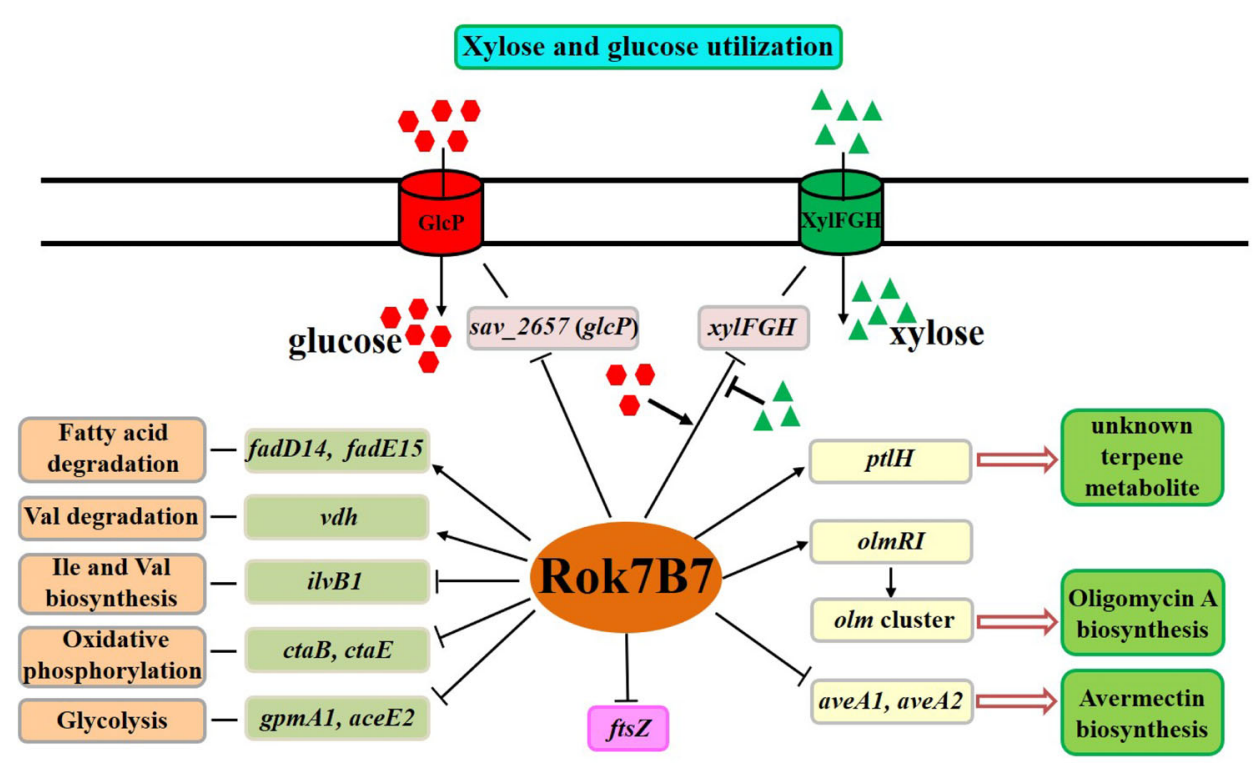

Fig. 10. Proposed schematic model of Rok7B7-mediated regulatory network in S. avermitilis. Bars: repression. Solid arrows: activation. Hollow arrows: production of secondary metabolites. Solid lines: involvement in primary metabolism. Triangles: xylose. Hexagons: glucose. [Color figure can be viewed at wileyonlinelibrary.com]
Primary metabolism

Secondary metabolism

and primers are listed in the Supporting Information Table S3. Growth conditions for E. coli and S. avermitilis were described previously (Jiang et al., 2011). YMS (lkeda et al., 1988) agar was used for phenotypic characterization of $S$. avermitilis mutants. Insoluble fermentation medium FM-I (Jiang et al., 2011) was used for avermectin and oligomycin A production, and soluble fermentation medium FM-II (Jiang et al., 2011) was used for quantitative analysis of $S$. avermitilis growth. Liquid MM (Kieser et al., 2000) supplemented with $10 \mathrm{mg} \mathrm{mL}^{-1}$ $(1 \% \mathrm{~W} / \mathrm{v})$ of various carbon sources was used for analysis of carbon uptake.

\section{Construction of S. avermitilis mutants}

For in-frame gene deletion of rok7B7, a 418-bp $5^{\prime}$ flanking region and a 379-bp $3^{\prime}$ flanking region were amplified from WT genome with respective primer pairs LXR1A/LXR1B and LXR2A/LXR2B. The two fragments were digested, respectively, with BamHI/Xbal and Xbal/ EcoRI and then ligated into BamHI/EcoRI-digested pKC1139 (Bierman et al., 1992), generating rok7B7 deletion vector pDrok7B7, which was transformed into WT protoplasts. rok7B7 deletion mutant $\Delta$ rok7B7 was screened as described previously (Yang et al., 2015), confirmed by PCR with primers LXR5A/LXR5B (flanking the exchange regions) and LXR6A/LXR6B (located within the deletion region) (Supporting Information Fig. S2) and subjected to DNA sequencing. To delete rok7B7 in industrial strain 63\#, vector pDrok7B7 was transformed into $63 \#$ protoplasts. The mutant, termed $\Delta$ rok7B7/63\#, was isolated by selection of $\Delta$ rok7B7 and confirmed by PCR with the same primers.

For complementation of $\Delta$ rok7B7, a 1565-bp DNA fragment carrying rok7B7 ORF and its promoter was amplified with primer pair LXR7A/LXR7B. The PCR product was cut out with $X b a l / E c o R I$ and inserted into pSET152 (Bierman et al., 1992) to obtain rok7B7-complemented vector pSET152-rok7B7, which was then introduced into $\Delta$ rok7B7 to obtain complemented strain Crok7B7.

For rok7B7 overexpression, a 1249-bp DNA fragment containing rok7B7 ORF was amplified with primers LXR4A/LXR4B, and ligated simultaneously with the 188-bp ermE* $p$ fragment from pJL117 (Li et al., 2010) into pKC1139 to produce rok7B7-overexpressing vector pKC-erm-rok7B7, which was then introduced into WT to generate rok7B7 overexpression strain Orok7B7.

To express $3 \times$ FLAG-tagged Rok7B7 in S. avermitilis, rok7B7 gene carrying its native promoter was amplified with primers $L X R 61 A / L X R 61 B$, and $3 \times$ FLAG fragment was amplified from plasmid plJ10500 (Pullan et al., 2011) with primers LXR60A/LXR60B. The resulting 1608-bp rok7B7 and 100-bp FLAG fragments were ligated into pSET152 to generate pSET152-rok7B7-3FLAG, which was then transformed into $\triangle$ rok7B7 to obtain recombinant strain $\Delta$ rok7B7/rok7B7-3FLAG for expression of C-terminally $3 \times$ FLAG-tagged Rok7B7.

\section{Scanning electron microscopy}

Streptomyces avermitilis WT and $\Delta$ rok7B7 strains were inoculated onto YMS plates and incubated at $28^{\circ} \mathrm{C}$ for 
2 or 4 days. Specimens were prepared and then observed by SEM as described previously (Sun et al., 2016).

\section{Fermentation and analysis of antibiotics}

Fermentation of $S$. avermitilis strains and quantitative analysis of avermectin and oligomycin A yield by HPLC were performed as described previously (Luo et al., 2014).

\section{Determination of sugar content}

Sugar content in fermentation broth was measured by HPLC. Fermentation broth $(1 \mathrm{ml})$ was centrifuged at $13000 \times g$ for $15 \mathrm{~min}$. The supernatant was filtered through nylon microporous (pore size $0.22 \mu \mathrm{m}$ ) membrane and applied to an HPLC system with ZORBAX Carbohydrate Analysis Column (5 $\mu \mathrm{m} ; 4.6 \times 250 \mathrm{~mm}$; Agilent; USA) and refractive index detector RID-20A (Shimadzu; Japan). Glucose, xylose, maltose, and mannitol were separated with acetonitrile/water $(80: 20, \mathrm{v} / \mathrm{v})$ at flow rate $1.0 \mathrm{ml} \mathrm{min}^{-1}$ and identified using respective standards.

\section{$q R T-P C R$ analysis}

Total RNAs were extracted at various time points from $S$. avermitilis cultures grown in liquid FM-I, in MM containing various sugars, or on YMS plates, using TRIzol reagent (Tiangen; China). Reverse transcription and subsequent real-time PCR assays were performed as described previously (Luo et al., 2014). Transcription levels of tested genes were determined by qRT-PCR using respective primers, with housekeeping gene 16S rRNA as internal control to normalize samples. Each experiment was performed in triplicate.

\section{Heterologous expression and purification of His $_{6}$-tagged Rok7B7 protein}

For heterologous expression of $S$. avermitilis Rok7B7 in E. coli, the 1263-bp rok7B7 coding region was amplified by PCR from genomic DNA of ATCC31267 using primer pair LXR3A/LXR3B. The PCR fragment was cut out with $\mathrm{BamHI} /$ EcoRI and cloned into pET-28a (+), generating expression vector $\mathrm{pET} 28$-rok7B7, which was verified by sequencing. 1256-bp rok7B7sco gene was similarly amplified using primer pair LXR62A/LXR62B from genomic DNA of S. coelicolor M145, and 1267-bp rok7B7sven gene was amplified using primer pair LXR63A/LXR63B from genomic DNA of $S$. venezuelae ISP5230. The two PCR fragments were ligated into pET-28a (+) to obtain pET28-rok7B7sco and pET28-rok7B7sven, respectively.
The three rok7B7 expression vectors were transformed separately into $E$. coli Rosetta (DE3), and expression of $\mathrm{N}$-terminal $\mathrm{His}_{6}$-tagged Rok7B7 recombinant protein was induced by addition of $0.2 \mathrm{mM}$ IPTG, followed by incubation for $3 \mathrm{~h}$ at $37^{\circ} \mathrm{C}$. Cells were harvested, resuspended in lysis buffer (Guo et al., 2018), sonicated on ice, and centrifuged. Soluble recombinant protein from supernatant was purified using a Ni-NTA column (Bio-works; Sweden), and fractions eluted with $500 \mathrm{mM}$ imidazole were dialyzed against binding buffer for EMSAs or HBSEP buffer for BLI assays to eliminate imidazole. Protein concentration was determined by Bradford assay, and purified recombinant protein was stored at $-80^{\circ} \mathrm{C}$.

\section{EMSAS}

EMSAs were performed as described previously (Sun et al., 2016). Promoter probes containing putative Rok7B7-binding sequences were generated by PCR using corresponding primers, and $3^{\prime}$-terminally labelled with nonradioactive digoxigenin-1-ddUTP. In binding reactions $(20 \mu \mathrm{l}), 0.3 \mathrm{nM}$ labelled probe was incubated with $1 \mu \mathrm{g}$ poly $[\mathrm{d}(\mathrm{I}-\mathrm{C})]$ and various amounts of $\mathrm{His}_{6}$ Rok7B7 at $25^{\circ} \mathrm{C}$ for $30 \mathrm{~min}$. Specificity of Rok7B7/probe interaction was confirmed by adding $\sim 300$-fold excess of unlabeled $h r d B p$ (nonspecific probe) or each specific probe to the reaction system.

For EMSAs with sugars, glucose or xylose was dissolved in deionized water and added to the reaction system at various final concentrations.

\section{Western blotting}

Western blotting was performed as described previously (Zhu et al., 2016). Total protein of $\Delta$ rok7B7/rok7B73FLAG was prepared from mycelia grown in FM-I for various durations. Anti-FLAG M2 mouse antibody (mAb) (Sigma; USA) was used at ratio 1:3300.

\section{ChIP-qPCR}

Mycelia of S. avermitilis WT and $\Delta$ rok7B7/rok7B7-3FLAG cultured in soluble FM-II for various times were collected, and processed as described previously (Yan et al., 2019). One millilitre input sample (2 mg protein $/ \mathrm{mL}$ ) was used for each immunoprecipitation, and $10 \mu \mathrm{l}$ was kept as input DNA. The ChIP reaction was performed with $1 \mu \mathrm{l}$ anti-FLAG $\mathrm{mAb}$, and the immunoprecipitated DNA was quantified by real-time PCR using primer pairs listed in the Supporting Information Table S3. The WT strain was used as negative control. ChIP-qPCR data were normalized by the ratio of ChIP sample to input DNAand expressed as a percentage of input DNA. The relative values of protein enrichment were calculated 
using the comparative $\mathrm{Ct}$ method: $\Delta \mathrm{Ct}(\mathrm{ChIP})=\mathrm{Ct}$ (ChIP)-(Ct (Input)-6.644); Input\% $=2^{-\Delta \mathrm{Ct}}($ ChIP) $\times 100 \%$. Each experiment was independently performed at least three times.

\section{5' RACE}

Transcriptional start sites (TSSs) of aveA1 and olmRI were determined by $5^{\prime}$ RACE using $5^{\prime} / 3^{\prime}$ RACE kit (Roche; USA). Total RNA $(2 \mu \mathrm{g})$ extracted from 48 -h culture of $S$. avermitilis WT grown in FM-I was used for reverse transcription with gene-specific primer sp1-aveA1 or sp1-olmRI (20 pmol). The obtained cDNAs were purified and treated with terminal transferase (TaKaRa; China) for addition of $3^{\prime}$ oligo(dA) tails. The tailed cDNA was PCR-amplified with oligo(dT) anchor primer and specific nested primer sp2-aveA1 or sp2-olmRI to yield a single specific band. The resulting PCR product was purified for sequencing, and TSS was mapped as the first base following oligo(dT) sequence.

\section{BLI assay}

BLI assay was performed using an Octet RED96 system (FortéBio; USA) to assess molecular interactions of Rok7B7 with its binding sequences. $5^{\prime}$ biotin-labelled 50-bp DNA fragments containing Rok7B7-binding sites were obtained by annealing primers listed in the Supporting Information Table S3. The reaction mixtures (each $200 \mu \mathrm{l}$ ) contained $300 \mathrm{nM}$ biotin-labelled DNA probes and various concentrations of purified $\mathrm{His}_{6}$-Rok7B7 in HBS-EP buffer [10 mM HEPES, $15 \mathrm{mM} \mathrm{NaCl}, 3 \mathrm{mM}$ EDTA, $0.005 \%(\mathrm{v} / \mathrm{v})$ Tween $20 \%$, and $0.1 \%(\mathrm{w} / \mathrm{v})$ BSA (pH 7.4)]. The assay procedure consisted of five steps: baseline, loading, washing, association, and dissociation. All steps were performed at $25^{\circ} \mathrm{C}$ in a 96-well plate containing $200 \mu \mathrm{l}$ reaction mixture and a streptavidin (SA) biosensor probe in each well. Blank tests, for purpose of baseline balance, were performed using HBS-EP buffer instead of $\mathrm{His}_{6}-$ Rok7B7 in association step. Data were processed using software programme Octet Analysis 21 CFR, Part 11 (V. 9.0).

To analyse effects of ligands on binding of Rok7B7 to its target sequences, glucose or xylose was added to the reaction system at indicated concentrations.

\section{Acknowledgements}

This study was supported by the National Key Research and Development Program of China (Grant No. 2017YFD0201207) and the National Natural Science Foundation of China (Grant No. 31872629). The authors are grateful to Dr. S. Anderson for English editing of the manuscript.

\section{References}

Bekiesch, P., Forchhammer, K., and Apel, A.K. (2016) Characterization of DNA binding sites of RokB, a ROK-family regulator from Streptomyces coelicolor reveals the RokB regulon. PLoS One 11: e0153249.

Bierman, M., Logan, R., O'Brien, K., Seno, E.T., Rao, R.N., and Schoner, B.E. (1992) Plasmid cloning vectors for the conjugal transfer of DNA from Escherichia coli to Streptomyces spp. Gene 116: 43-49.

Burg, R.W., Miller, B.M., Baker, E.E., Birnbaum, J., Currie, S.A., Hartman, R., et al. (1979) Avermectins, new family of potent anthelmintic agents: producing organism and fermentation. Antimicrob Agents Chemother 15: 361-367.

Bush, M.J., Chandra, G., Bibb, M.J., Findlay, K.C., and Buttner, M.J. (2016) Genome-wide chromatin immunoprecipitation sequencing analysis shows that WhiB is a transcription factor that cocontrols its regulon with WhiA to initiate developmental cell division in Streptomyces. mBio 7: e00523-e00516.

Chen, S., Zheng, G., Zhu, H., He, H., Chen, L., Zhang, W., et al. (2016) Roles of two-component system AfsQ1/Q2 in regulating biosynthesis of the yellow-pigmented coelimycin P2 in Streptomyces coelicolor. FEMS Microbiol Lett 363: fnw160.

Chen, Y., Wendt-Pienkowski, E., and Shen, B. (2008) Identification and utility of FdmR1 as a Streptomyces antibiotic regulatory protein activator for fredericamycin production in Streptomyces griseus ATCC 49344 and heterologous hosts. J Bacteriol 190: 5587-5596.

Chng, C., Lum, A.M., Vroom, J.A., and Kao, C.M. (2008) A key developmental regulator controls the synthesis of the antibiotic erythromycin in Saccharopolyspora erythraea. Proc Natl Acad Sci U S A 105: 11346-11351.

Dedrick, R.M., Wildschutte, H., and McCormick, J.R. (2009) Genetic interactions of $s m c$, fts $K$, and parB genes in Streptomyces coelicolor and their developmental genome segregation phenotypes. J Bacteriol 191: 320-332.

Dubeau, M.P., Poulin-Laprade, D., Ghinet, M.G., and Brzezinski, R. (2011) Properties of CsnR, the transcriptional repressor of the chitosanase gene, $\operatorname{csnA}$, of Streptomyces lividans. J Bacteriol 193: 2441-2450.

Egerton, J.R., Ostlind, D.A., Blair, L.S., Eary, C.H., Suhayda, D., Cifelli, S., et al. (1979) Avermectins, new family of potent anthelmintic agents: efficacy of the B1a component. Antimicrob Agents Chemother 15: 372-378.

Flardh, K., and Buttner, M.J. (2009) Streptomyces morphogenetics: dissecting differentiation in a filamentous bacterium. Nat Rev Microbiol 7: 36-49.

Guo, J., Zhang, X., Lu, X., Liu, W., Chen, Z., Li, J., et al. (2018) SAV4189, a MarR-family regulator in Streptomyces avermitilis, activates avermectin biosynthesis. Front Microbiol 9: 1358.

Guo, J., Zhao, J., Li, L., Chen, Z., Wen, Y., and Li, J. (2010) The pathway-specific regulator AveR from Streptomyces avermitilis positively regulates avermectin production while it negatively affects oligomycin biosynthesis. Mol Genet Genomics 283: 123-133.

Hiard, S., Maree, R., Colson, S., Hoskisson, P.A., Titgemeyer, F., van Wezel, G.P., et al. (2007) PREDetector: a new tool to 
identify regulatory elements in bacterial genomes. Biochem Biophys Res Commun 357: 861-864.

Hindle, Z., and Smith, C.P. (1994) Substrate induction and catabolite repression of the Streptomyces coelicolor glycerol operon are mediated through the GyIR protein. $\mathrm{Mol}$ Microbiol 12: 737-745.

Ikeda, H., Kotaki, H., Tanaka, H., and Omura, S. (1988) Involvement of glucose catabolism in avermectin production by Streptomyces avermitilis. Antimicrob Agents Chemother 32: 282-284.

Ikeda, H., Nonomiya, T., Usami, M., Ohta, T., and Omura, S. (1999) Organization of the biosynthetic gene cluster for the polyketide anthelmintic macrolide avermectin in Streptomyces avermitilis. Proc Natl Acad Sci U S A 96: 9509-9514.

Ikeda, H., and Omura, S. (1997) Avermectin biosynthesis. Chem Rev 97: 2591-2610.

Jiang, L., Liu, Y., Wang, P., Wen, Y., Song, Y., Chen, Z., and $\mathrm{Li}, \mathrm{J}$. (2011) Inactivation of the extracytoplasmic function sigma factor $\sigma^{6}$ stimulates avermectin production in Streptomyces avermitilis. Biotechnol Lett 33: 1955-1961.

Kazanov, M.D., Li, X., Gelfand, M.S., Osterman, A.L., and Rodionov, D.A. (2013) Functional diversification of ROKfamily transcriptional regulators of sugar catabolism in the Thermotogae phylum. Nucleic Acids Res 41: 790-803.

Keijser, B.J., Noens, E.E., Kraal, B., Koerten, H.K., and van Wezel, G.P. (2003) The Streptomyces coelicolor ssgB gene is required for early stages of sporulation. FEMS Microbiol Lett 225: 59-67.

Kieser, T., Bibb, M.J., Buttner, M.J., Chater, K.F., and Hopwood, D.A. (2000) Practical Streptomyces Genetics, Norwich, UK: The John Innes Foundation.

Kitani, S., Ikeda, H., Sakamoto, T., Noguchi, S., and Nihira, T. (2009) Characterization of a regulatory gene, ave $R$, for the biosynthesis of avermectin in Streptomyces avermitilis. Appl Microbiol Biotechnol 82: 1089-1096.

Li, L., Guo, J., Wen, Y., Chen, Z., Song, Y., and Li, J. (2010) Overexpression of ribosome recycling factor causes increased production of avermectin in Streptomyces avermitilis strains. $J$ Ind Microbiol Biotechnol 37: 673-679.

Liguori, R., Ventorino, V., Pepe, O., and Faraco, V. (2016) Bioreactors for lignocellulose conversion into fermentable sugars for production of high added value products. Appl Microbiol Biotechnol 100: 597-611.

Lin, X., Wen, Y., Li, M., Chen, Z., Guo, J., Song, Y., and Li, J. (2009) A new strain of Streptomyces avermitilis produces high yield of oligomycin A with potent anti-tumor activity on human cancer cell lines in vitro. Appl Microbiol Biotechnol 81: 839-845.

Liu, S.P., Yu, P., Yuan, P.H., Zhou, Z.X., Bu, Q.T., Mao, X.M., and Li, Y.Q. (2015) Sigma factor WhiGch positively regulates natamycin production in Streptomyces chattanoogensis L10. Appl Microbiol Biotechnol 99: 2715-2726.

Luo, S., Sun, D., Zhu, J., Chen, Z., Wen, Y., and Li, J. (2014) An extracytoplasmic function sigma factor, $\sigma^{25}$, differentially regulates avermectin and oligomycin biosynthesis in Streptomyces avermitilis. Appl Microbiol Biotechnol 98: 7097-7112.

Mao, X.M., Luo, S., Zhou, R.C., Wang, F., Yu, P., Sun, N., et al. (2015) Transcriptional regulation of the daptomycin gene cluster in Streptomyces roseosporus by an autoregulator, AtrA. J Biol Chem 290: 7992-8001.

Nguyen, J., Francou, F., Virolle, M.J., and Guerineau, M. (1997) Amylase and chitinase genes in Streptomyces lividans are regulated by reg1, a pleiotropic regulatory gene. J Bacteriol 179: 6383-6390.

$\mathrm{Ni}, \mathrm{X}$., and Westpheling, J. (1997) Direct repeat sequences in the Streptomyces chitinase-63 promoter direct both glucose repression and chitin induction. Proc Natl Acad Sci U S A 94: 13116-13121.

Noguchi, Y., Kashiwagi, N., Uzura, A., Ogino, C., Kondo, A., Ikeda, H., and Sota, M. (2018) Development of a strictly regulated xylose-induced expression system in Streptomyces. Microb Cell Fact 17: 151.

Omura, S., Ikeda, H., Ishikawa, J., Hanamoto, A., Takahashi, C., Shinose, M., et al. (2001) Genome sequence of an industrial microorganism Streptomyces avermitilis: deducing the ability of producing secondary metabolites. Proc Natl Acad Sci U S A 98: 12215-12220.

Pennacchio, A., Ventorino, V., Cimini, D., Pepe, O., Schiraldi, C., Inverso, M., and Faraco, V. (2018) Isolation of new cellulase and xylanase producing strains and application to lignocellulosic biomasses hydrolysis and succinic acid production. Bioresour Technol 259: 325-333.

Pinna, L.A., Lorini, M., Moret, V., and Siliprandi, N. (1967) Effect of oligomycin and succinate on mitochondrial metabolism of adenine nucleotides. Biochim Biophys Acta 143: $18-25$.

Plumbridge, J. (2001) Regulation of PTS gene expression by the homologous transcriptional regulators, Mlc and NagC, in Escherichia coli (or how two similar repressors can behave differently). J Mol Microbiol Biotechnol 3: 371-380.

Pullan, S.T., Chandra, G., Bibb, M.J., and Merrick, M. (2011) Genome-wide analysis of the role of GInR in Streptomyces venezuelae provides new insights into global nitrogen regulation in actinomycetes. BMC Genomics 12: 175.

Romero-Rodriguez, A., Robledo-Casados, I., and Sanchez, S. (2015) An overview on transcriptional regulators in Streptomyces. Biochim Biophys Acta 1849: 1017-1039.

Romero-Rodriguez, A., Rocha, D., Ruiz-Villafan, B., Guzman-Trampe, S., Maldonado-Carmona, N., VazquezHernandez, M., et al. (2017) Carbon catabolite regulation in Streptomyces: new insights and lessons learned. World J Microbiol Biotechnol 33: 162.

Schwedock, J., McCormick, J.R., Angert, E.R., Nodwell, J. R., and Losick, R. (1997) Assembly of the cell division protein FtsZ into ladder-like structures in the aerial hyphae of Streptomyces coelicolor. Mol Microbiol 25: 847-858.

Sun, D., Zhu, J., Chen, Z., Li, J., and Wen, Y. (2016) SAV742, a novel AraC-family regulator from Streptomyces avermitilis, controls avermectin biosynthesis, cell growth and development. Sci Rep 6: 36915.

Swiatek, M.A., Gubbens, J., Bucca, G., Song, E., Yang, Y. H., Laing, E., et al. (2013) The ROK family regulator Rok7B7 pleiotropically affects xylose utilization, carbon catabolite repression, and antibiotic production in Streptomyces coelicolor. J Bacteriol 195: 1236-1248.

Swiatek-Polatynska, M.A., Bucca, G., Laing, E., Gubbens, J., Titgemeyer, F., Smith, C.P., et al. (2015) 
Genome-wide analysis of in vivo binding of the master regulator DasR in Streptomyces coelicolor identifies novel non-canonical targets. PLoS One 10: e0122479.

Tanaka, A., Takano, Y., Ohnishi, Y., and Horinouchi, S. (2007) AfsR recruits RNA polymerase to the afsS promoter: a model for transcriptional activation by SARPs. J Mol Biol 369: 322-333.

van Wezel, G.P., Mahr, K., Konig, M., Traag, B.A., PimentelSchmitt, E.F., Willimek, A., and Titgemeyer, F. (2005) GlcP constitutes the major glucose uptake system of Streptomyces coelicolor A3(2). Mol Microbiol 55: 624-636.

van Wezel, G.P., and McDowall, K.J. (2011) The regulation of the secondary metabolism of Streptomyces: new links and experimental advances. Nat Prod Rep 28: 1311-1333.

van Wezel, G.P., White, J., Young, P., Postma, P.W., and Bibb, M.J. (1997) Substrate induction and glucose repression of maltose utilization by Streptomyces coelicolor $\mathrm{A} 3(2)$ is controlled by malR, a member of the Lacl-GalR family of regulatory genes. Mol Microbiol 23: 537-549.

Vicente, C.M., Santos-Aberturas, J., Payero, T.D., Barreales, E.G., de Pedro, A., and Aparicio, J.F. (2014) PAS-LuxR transcriptional control of filipin biosynthesis in S. avermitilis. Appl Microbiol Biotechnol 98: 9311-9324.

Walter, S., and Schrempf, H. (1996) The synthesis of the Streptomyces reticuli cellulase (avicelase) is regulated by both activation and repression mechanisms. Mol Gen Genet 251: 186-195.

Willemse, J., Borst, J.W., de Waal, E., Bisseling, T., and van Wezel, G.P. (2011) Positive control of cell division: FtsZ is recruited by SsgB during sporulation of Streptomyces. Genes Dev 25: 89-99.
Yan, H., Lu, X., Sun, D., Zhuang, S., Chen, Q., Chen, Z., et al. (2019) BldD, a master developmental repressor, activates antibiotic production in two Streptomyces species. Mol Microbiol 113, 123-142.

Yang, R., Liu, X., Wen, Y., Song, Y., Chen, Z., and Li, J. (2015) The PhoP transcription factor negatively regulates avermectin biosynthesis in Streptomyces avermitilis. Appl Microbiol Biotechnol 99: 10547-10557.

Yin, S., Wang, W., Wang, X., Zhu, Y., Jia, X., Li, S., et al. (2015) Identification of a cluster-situated activator of oxytetracycline biosynthesis and manipulation of its expression for improved oxytetracycline production in Streptomyces rimosus. Microb Cell Fact 14: 46.

Yu, Q., Bai, L.Q., Zhou, X.F., and Deng, Z.X. (2012) Inactivation of the positive LuxR-type oligomycin biosynthesis regulators OImRI and OImRII increases avermectin production in Streptomyces avermitilis. Chin Sci Bull 8: 869-876.

Zhu, J., Sun, D., Liu, W., Chen, Z., Li, J., and Wen, Y. (2016) AvaR2, a pseudo gamma-butyrolactone receptor homologue from Streptomyces avermitilis, is a pleiotropic repressor of avermectin and avenolide biosynthesis and cell growth. Mol Microbiol 102: 562-578.

\section{Supporting Information}

Additional Supporting Information may be found in the online version of this article at the publisher's web-site:

Appendix S1: Supporting information 